\title{
Global Matrix 3.0 Physical Activity Report Card Grades for Children and Youth: Results and Analysis From 49 Countries
}

\author{
Salomé Aubert, Joel D. Barnes, Chalchisa Abdeta, Patrick Abi Nader, Ade F. Adeniyi, Nicolas \\ Aguilar-Farias, Dolores S. Andrade Tenesaca, Jasmin Bhawra, Javier Brazo-Sayavera, Greet \\ Cardon, Chen-Kang Chang, Christine Delisle Nyström, Yolanda Demetriou, Catherine E. Draper, \\ Lowri Edwards, Arunas Emeljanovas, Aleš Gába, Karla I. Galaviz, Silvia A. González, Marianella \\ Herrera-Cuenca, Wendy Y. Huang, Izzeldin A.E. Ibrahim, Jaak Jürimäe, Katariina Kämppi, Tarun \\ R. Katapally, Piyawat Katewongsa, Peter T. Katzmarzyk, Asaduzzaman Khan, Agata Korcz, Yeon \\ Soo Kim, Estelle Lambert, Eun-Young Lee, Marie Löf, Tom Loney, Juan López-Taylor, Yang Liu, \\ Daga Makaza, Taru Manyanga, Bilyana Mileva, Shawnda A. Morrison, Jorge Mota, Vida K. \\ Nyawornota, Reginald Ocansey, John J. Reilly, Blanca Roman-Viñas, Diego Augusto Santos \\ Silva, Pairoj Saonuam, John Scriven, Jan Seghers, Natasha Schranz, Thomas Skovgaard, Melody \\ Smith, Martyn Standage, Gregor Starc, Gareth Stratton, Narayan Subedi, Tim Takken, Tuija \\ Tammelin, Chiaki Tanaka, David Thivel, Dawn Tladi, Richard Tyler, Riaz Uddin, Alun Williams, \\ Stephen H.S. Wong, Ching-Lin Wu, Paweł Zembura, and Mark S. Tremblay
}

Background: Accumulating sufficient moderate to vigorous physical activity is recognized as a key determinant of physical, physiological, developmental, mental, cognitive, and social health among children and youth (aged 5-17 y). The Global Matrix 3.0 of Report Card grades on physical activity was developed to achieve a better understanding of the global variation in child and youth physical activity and associated supports. Methods: Work groups from 49 countries followed harmonized procedures to develop their Report Cards by grading 10 common indicators using the best available data. The participating countries were divided into 3 categories using the United Nations' human development index (HDI) classification (low or medium, high, and very high HDI). Results: A total of 490 grades, including 369 letter grades and 121 incomplete grades, were assigned by the 49 work groups. Overall, an average grade of "C-," "D+," and "C-" was obtained for the low and medium HDI countries, high HDI countries, and very high HDI countries, respectively. Conclusions: The present study provides rich new evidence showing that the situation regarding the physical activity of children and youth is a concern worldwide. Strategic public investments to implement effective interventions to increase physical activity opportunities are needed.

Keywords: global comparison, sedentary behavior, health promotion, international, sport, active transportation

Physical inactivity, defined as engaging in insufficient levels of physical activity and not meeting the current physical activity recommendations, ${ }^{1}$ has been identified as the fourth leading risk factor of premature mortality in adulthood..$^{2}$ In contrast, accumulating sufficient moderate- to vigorous-intensity physical activity (MVPA) is recognized as a key determinant of physical, mental,

Aubert, Barnes, Delisle Nyström, González, Manyanga, and Tremblay are with Healthy Active Living and Obesity Research Group, CHEO Research Institute, Ottawa, Ontario, Canada. Abdeta is with Hiwot Fana Specialized Hospital, Haramaya University, Harar, Ethiopia. Abi Nader is with the Centre de formation médicale du NouveauBrunswick, Université de Moncton, Moncton, New Brunswick, Canada; and Centre de Recherche du Centre Hospitalier de l'Université de Montréal, Montréal, Québec, Canada. Adeniyi is with College of Medicine, University of Ibadan, Ibadan, Nigeria; and Nigerian Heart Foundation, Lagos, Nigeria. Aguilar-Farias is with the Department of Physical Education, Sports and Recreation, Universidad de La Frontera, Temuco, Chile; and UFRO Actívate Research Group, Universidad de La Frontera, Temuco, Chile. Andrade Tenesaca is with the Departamento de Biociencias, Universidad de Cuenca, Cuenca, Ecuador. Bhawra is with the School of Public Health and Health Systems, University of Waterloo, Waterloo, Ontario, Canada. Brazo-Sayavera is with the Instituto Superior de Educación Física, Universidad de la República, Rivera, Uruguay; and the Polos de Desarrollo Universitario EFISAL, Universidad de la República, Rivera, Uruguay. Cardon is with the Department of Movement and Sports Sciences, Ghent University, Ghent, Belgium. Chang is with the Department of Sport Performance, National Taiwan University of Sport, Taichung, Taiwan. Delisle Nyström is also with the Department of Biosciences and Nutrition, Karolinska Institutet, Huddinge, Sweden. Demetriou is with the Department of Sport and Health Sciences, Technical University of Munich, Munich, Germany. Draper is with the University of the Witwatersrand, Johannesburg, South Africa; and the University of Cape Town, Cape Town, South Africa. Edwards, Stratton, and Tyler are with the Applied Sport Technology Exercise and Medicine Research Centre, Swansea University, Swansea, Wales, United Kingdom. Emeljanovas is with Lithuanian Sports University, Kaunas, Lithuania. Gába is with the Faculty of Physical Culture, Palacký University Olomouc, Olomouc, Czech Republic. Galaviz is with Emory Global Diabetes Research Center, Hubert Department of Global Health, Rollins School of Public Health, Emory University, Atlanta, GA, USA. González is also with the School of Medicine, Universidad de los Andes, Bogotá, Colombia. Herrera-Cuenca is with the Centro de Estudios del Desarrollo, Universidad Central de Venezuela, Caracas, Venezuela; Fundación Bengoa para la Alimentación y Nutrición, Caracas, Venezuela; and Observatorio Venezolano de la Salud, Caracas, Venezuela. Huang is with the Department of Sport and Physical Education, Hong Kong Baptist University, Hong Kong, China. Ibrahim is with Aspetar Orthopedic and Sports Medicine Hospital, Doha, Qatar. Jürimäe is with the Institute of Sport Sciences and Physiotherapy, Faculty of Medicine, University of Tartu, Tartu, Estonia. Kämppi and Tammelin are with the LIKES Research Centre for Physical Activity and Health, Jyväskylä, Finland. Katapally is with Johnson Shoyama Graduate School of Public Policy, University of Regina, Regina, Saskatchewan, Canada. Katewongsa is with the Institute for Population and Social Research, Mahidol University, Salaya, 
social, and environmental health. ${ }^{3,4}$ Among children and youth (aged 5-17 y), several systematic reviews have reported physical activity benefits on physical, physiological, developmental, mental, cognitive, and social health, as well as academic achievement. ${ }^{5-}$ ${ }^{10}$ Despite these benefits, it has been estimated that $80 \%$ of youth (11-17 y old) worldwide do not reach the minimum recommendation of 60 minutes of MVPA per day. ${ }^{11}$ This is alarming given that physical inactivity among school-aged children and youth has been found to be associated with adverse physical, mental, social, and cognitive health outcomes, $, 5,8,12,13$ lower physical fitness, ${ }^{14}$ and lower physical activity levels in later life. ${ }^{15}$

To achieve a better understanding of the global variation in child and youth physical activity and its correlates, the Global Matrix of Report Card grades on physical activity was launched for the first time in 2014. ${ }^{16}$ Physical activity Report Cards were developed based on the Canadian Report Card model, ${ }^{17}$ using a harmonized process for data gathering, assessing, and assigning grades to indicators. For over a decade, the Canadian Report Card has been successful in raising awareness and influencing policies for childhood physical activity promotion. However, these efforts have not yet translated into improving the physical activity levels of Canadian children and youth. ${ }^{18}$ Fifteen countries in 2014 (Global Matrix 1.0) and 38 countries in 2016 (Global Matrix 2.0) developed and launched Report Cards presenting grades for 9 physical activity indicators, allowing for international comparisons and offering insights from global data. ${ }^{16,19}$ The first 2 Global Matrices enabled the identification of several gaps in surveillance and research practice. Also, a paradox of higher physical activity and lower sedentary behavior in countries reporting poorer infrastructure, and lower physical activity and higher sedentary behavior in countries reporting better infrastructure was highlighted. ${ }^{16,19}$ Although participation in the Global Matrices 1.0 and 2.0 facilitated capacity building, professional networking, research collaborations, and international comparisons, the Global Matrix framework still needs to be continuously expanded, improved, replicated, and widely disseminated. ${ }^{19}$

The Global Matrix initiative is led by the Active Healthy Kids Global Alliance (AHKGA), which is an incorporated not-for-profit organization consisting of researchers, health professionals, and stakeholders who collaborate to advance physical activity in children and youth from around the world. ${ }^{20}$ In 2017, the AHKGA invited previous participating countries and called for new countries to register for the Global Matrix 3.0. As a result, work groups from 49 countries completed the full registration process and followed the harmonized procedures to develop their Report Cards by grading 10 common indicators (Overall Physical Activity, Organized Sport and Physical Activity, Active Play, Active Transportation, Sedentary Behaviors, Physical Fitness, Family and Peers, School, Community and Environment, and Government) using the best available data.

Countries in different development stages may have different challenges and priorities to inform strategies to improve physical activity among children and youth. Countries involved in the Global Matrix 3.0 were classified within 3 categories using the human development index (HDI): low and medium HDI $(<0.70)$, high HDI $(\geq 0.70$ to $<0.80)$, and very high HDI $(\geq 0.80)$. The HDI, ranging from 0 to 1 , is a composite index calculated using data on education, life expectancy, and income per capita, ${ }^{21}$ and was created by the United Nations Development Programme to rank countries on a scale of human development conceptualized in terms of capabilities of humans within the countries to function. ${ }^{22}$ Nine of the participating countries were classified as low or medium HDI (Bangladesh, Botswana, Ethiopia, Ghana, India, Nepal, Nigeria, South Africa, and Zimbabwe), 10 as high HDI (Brazil, Bulgaria, China, Colombia, Ecuador, Lebanon, Mexico, Thailand, Uruguay, and Venezuela), and 30 countries and territories as very high HDI

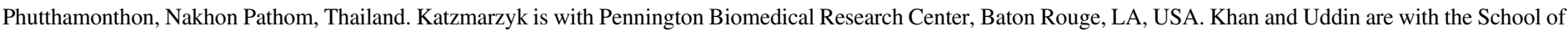



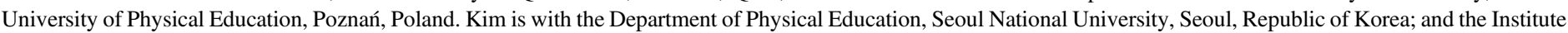

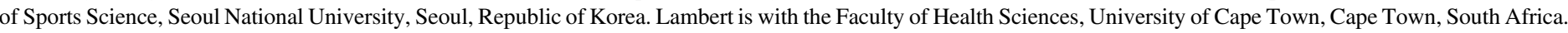

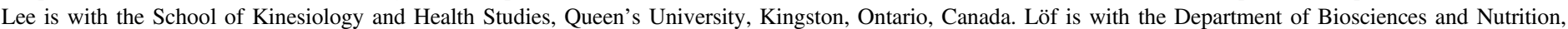

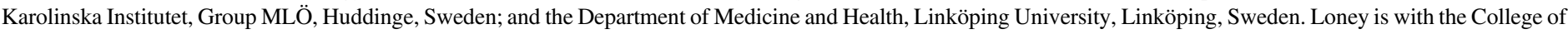

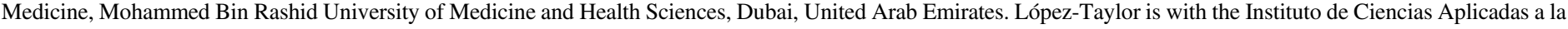

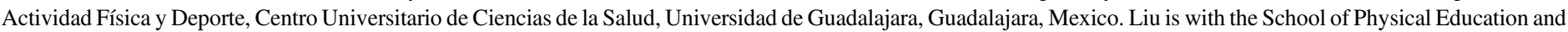

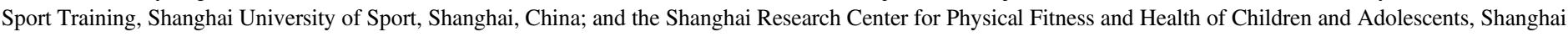

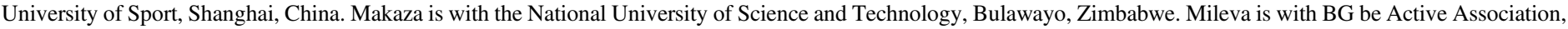

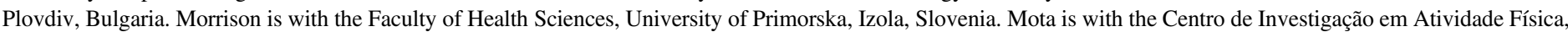

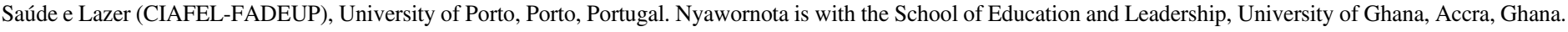

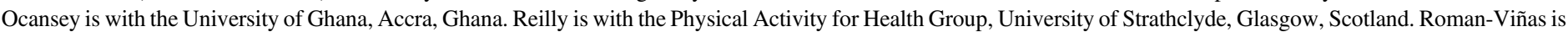

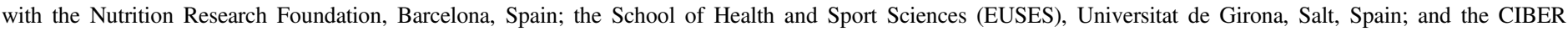

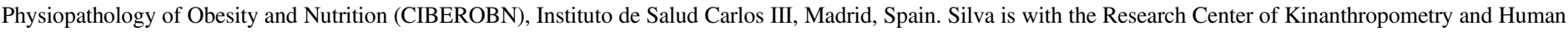

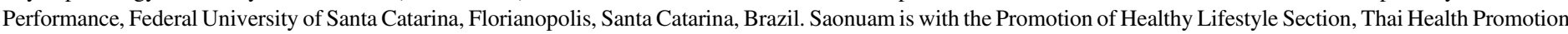

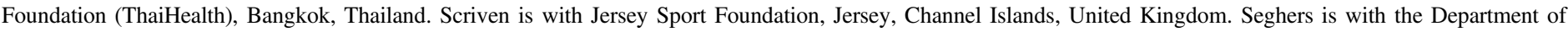

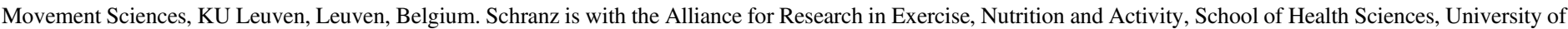

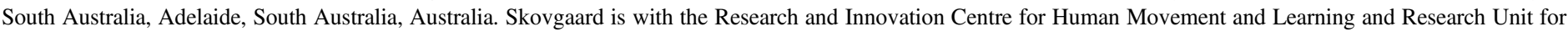

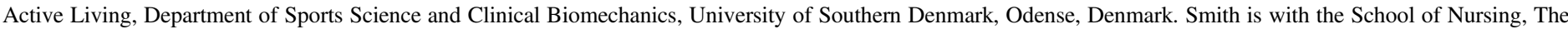



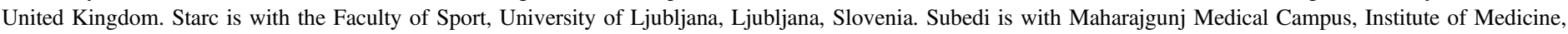

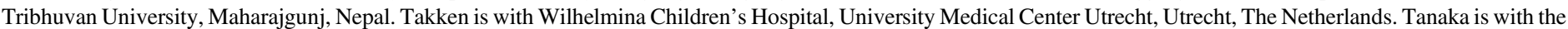

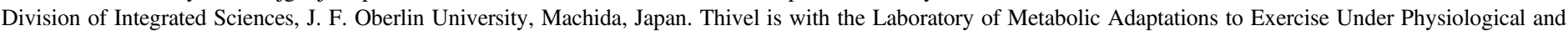



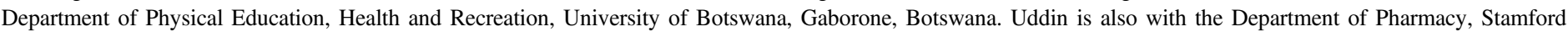

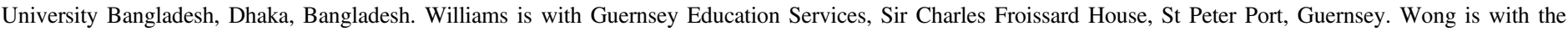

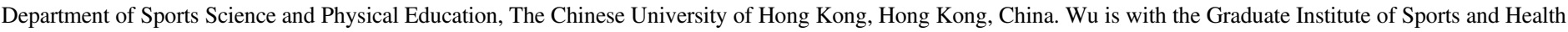

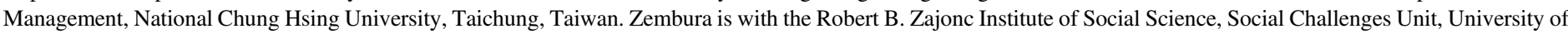
Warsaw, Warsaw, Poland. Tremblay (mtremblay@cheo.on.ca) is corresponding author. 
(Australia, Belgium [Flanders], Canada, Chile, Chinese Taipei [Taiwan], Czech Republic, Denmark, England, Estonia, Finland, France, Germany, Guernsey, Hong Kong, Japan, Jersey, Lithuania, The Netherlands, New Zealand, Poland, Portugal, Qatar, Scotland, Slovenia, South Korea, Spain, Sweden, United Arab Emirates, United States, and Wales).

This special issue of the Journal of Physical Activity and Health includes 49 extended abstracts documenting the main Report Card findings from each participating country. In addition to this current paper, 3 papers were developed to present and discuss results from the Report Cards by predetermined HDI categories as follows:

- "The Indicators of Physical Activity among Children and Youth in Nine Countries with Low and Medium Human Development Indices: A Global Matrix 3.0 Paper"23;

- "Report Card Grades on the Physical Activity of Children and Youth from 10 Countries with High Human Development Index-Global Matrix 3.0"24;

- "Report Card Grades on the Physical Activity of Children and Youth Comparing 30 Very High Human Development Index Countries." 25

The aim of this integrated article is to combine and compare the findings from each of the HDI (low and medium, high, and very high) papers and present, compare, and discuss further analyses of the results from the 49 countries participating in the Global Matrix 3.0.

\section{Methods}

\section{Creating the Global Matrix 3.0}

In April 2017, the AHKGA invited interested countries to participate in and register for the Global Matrix 3.0 through an open call that was distributed via established networks. Between April 2017 and January 2018, 49 countries from 6 different continents (Africa, Asia, Europe, North America, Oceania, and South America) registered and paid a registration fee based on their HDI classification to cover costs associated with the Global Matrix 3.0 initiative. Three different tiers of registration fees $\mathbf{\$ 5 0 0}$ USD for the low HDI countries, $\$ 750$ USD for the medium HDI countries, $\$ 1000$ USD for the high HDI countries, and \$1500 USD for the very high HDI countries) were offered to encourage equitable participation from around the world. Individuals who registered on behalf of their country were responsible for leading the effort to form a multidisciplinary Report Card work group of national physical activity experts and stakeholders. Furthermore, 1 to 3 Report Card leaders/coleaders per country were designated officially to manage the national Report Card development, and to ensure effective communication between the AHKGA Executive Committee and the Report Card work group.

A mentoring system was developed for the Global Matrix 3.0. New Report Card leaders and teams were paired with an experienced Report Card leader from a country who participated in the Global Matrix 2.0. In addition, 6 members of the Executive Committee of AHKGA were assigned to be regional mentors for each involved continent to provide help and guidance to the countries when needed and report their progress to the AHKGA Executive Committee. Finally, the AHKGA Executive Committee served as the coordinating center of the Global Matrix development and provided information including background papers, previous Report Cards and Report Card papers, fundraising suggestions, and a theoretical framework to support the preparation of grant proposals, scholarship, and funding applications. The AHKGA Executive Committee also provided guidance through monthly e-blasts by sharing milestones and upcoming deadlines.

\section{Harmonized Report Card Development}

An updated list of indicators (to which grades would be assigned) and corresponding benchmarks were created based on the previous Global Matrix methods, ${ }^{19}$ and feedback received during a workshop after the Global Matrix 2.0 launch in Bangkok, Thailand (November, 2016). A new indicator, Physical Fitness, and its corresponding benchmarks were added to the list. The benchmark for Overall Physical Activity was modified in accordance with the new Canadian 24-Hour Movement Guidelines for Children and Youth, ${ }^{26}$ where "at least 60 minutes of MVPA per day" was changed to "at least 60 minutes of MVPA per day on average." Further modifications to the benchmarks were made during the development of the Global Matrix 3.0 to address several issues that were reported by Report Card leaders to the AHKGA Executive Committee. The final version of the benchmarks for the 10 indicators is presented in Table 1. A more detailed grading scheme using positive $(+)$ and negative $(-)$ mathematical symbols was also developed (Table 2). In some countries, because of the modifications of the benchmarks from previous Global Matrices, grades for some indicators have changed while in fact, the situation was relatively the same. Consequently, while the Report Card work groups reported grades based on the revised benchmarks for the Global Matrix 3.0, some countries reported different grades in their national Report Grades (ie, to be consistent with their previous methods). For example, in Colombia, with the new benchmark, a "D+" was assigned to Overall Physical Activity for the Global Matrix 3.0, whereas a "D-" is reported in the Colombian country Report Card, based on the previous benchmark. ${ }^{28}$

Each country work group identified and complemented the list of indicators to be graded (ie, the 10 common indicators and potentially additional ones that would be included in their national Report Card but not in the Global Matrix 3.0) and gathered the highest quality of published and unpublished evidence, or in some cases, collected data prospectively. Due to the lack of data concerning physical activity among children in the early years $(0-4$ y) observed in the previous Global Matrices, consensus was reached among the AHKGA Executive Committee that the 10 indicator grades should only be informed by data from school-aged children and youth $(\sim 5-17$ y old $)$ for the Global Matrix 3.0 to ensure consistency across countries. Where possible, countries were also advised to consider and synthesize the best available evidence from approximately the past 5 years for each indicator. Through a harmonized and transparent Report Card development process, each country's work group collected and collated the best available evidence from local, national, or international studies, national surveys, official reports, and normative documents, and then synthesized findings and reached consensus for the grading of each indicator. A draft of each country Report Card grades were submitted along with their rationale by Report Card leaders and were audited by members of the AHKGA Executive Committee to ensure that the grades were consistent with the harmonized benchmarks and grading scheme. This audit process led to minor changes of the grades or rationale for most of the countries. 
Table 1 Global Matrix 3.0 Indicators and Benchmarks Used to Guide the Grade Assignment Process

\begin{tabular}{ll}
\hline Indicator & Benchmark \\
\hline Overall Physical & $\begin{array}{l}\% \text { of children and youth who meet the Global Recommendations on Physical Activity for Health, which recommend that } \\
\text { children and youth accumulate at least } 60 \text { min of moderate- to vigorous-intensity physical activity per day on average. }\end{array}$ \\
Activity & $\begin{array}{l}\text { Or \% of children and youth meeting the guidelines on at least } 4 \text { d a week (when an average cannot be estimated). } \\
\text { Organized Sport and }\end{array} \%$ of children and youth who participate in organized sport and/or physical activity programs.
\end{tabular}

Physical Activity

Active Play

$\%$ of children and youth who engage in unstructured/unorganized active play at any intensity for more than $2 \mathrm{~h}$ a day. $\%$ of children and youth who report being outdoors for more than $2 \mathrm{~h}$ a day.

Active Transportation $\%$ of children and youth who use active transportation to get to and from places (eg, school, park, mall, friend's house).

Sedentary Behaviors $\quad \%$ of children and youth who meet the Canadian Sedentary Behaviour Guidelines (5- to 17-y-olds: no more than 2 h of recreational screen time per day). Note: the Guidelines currently provide a time limit recommendation for screen-related pursuits, but not for nonscreen-related pursuits.

Physical Fitness Average percentile achieved on certain physical fitness indicators based on the normative values published by Tomkinson et al. 27

Family and Peers $\quad \%$ of family members (eg, parents, guardians) who facilitate physical activity and sport opportunities for their children (eg, volunteering, coaching, driving, paying for membership fees and equipment).

$\%$ of parents who meet the Global Recommendations on Physical Activity for Health, which recommend that adults accumulate at least $150 \mathrm{~min}$ of moderate-intensity aerobic physical activity throughout the week or do at least 75 min of vigorous-intensity aerobic physical activity throughout the week or an equivalent combination of moderate- and vigorousintensity physical activity.

$\%$ of family members (eg, parents, guardians) who are physically active with their kids.

$\%$ of children and youth with friends and peers who encourage and support them to be physically active.

$\%$ of children and youth who encourage and support their friends and peers to be physically active.

School $\%$ of schools with active school policies (eg, daily physical education (PE), daily physical activity, recess, "everyone plays" approach, bike racks at school, traffic calming on school property, outdoor time).

$\%$ of schools where the majority $(\geq 80 \%)$ of students are taught by a PE specialist.

$\%$ of schools where the majority $(\geq 80 \%)$ of students are offered the mandated amount of PE (for the given state/territory/ region/country).

$\%$ of schools that offer physical activity opportunities (excluding PE) to the majority $(>80 \%)$ of their students.

$\%$ of parents who report their children and youth have access to physical activity opportunities at school in addition to PE classes.

$\%$ of schools with students who have regular access to facilities and equipment that support physical activity (eg, gymnasium, outdoor playgrounds, sporting fields, multipurpose space for physical activity, equipment in good condition).

Community and Environment

$\%$ of children or parents who perceive their community/municipality is doing a good job at promoting physical activity (eg, variety, location, cost, quality).

$\%$ of communities/municipalities that report they have policies promoting physical activity.

$\%$ of communities/municipalities that report they have infrastructure (eg, sidewalks, trails, paths, bike lanes) specifically geared toward promoting physical activity.

$\%$ of children or parents who report having facilities, programs, parks, and playgrounds available to them in their community. $\%$ of children or parents who report living in a safe neighborhood where they can be physically active.

$\%$ of children or parents who report having well-maintained facilities, parks, and playgrounds in their community that are safe to use.

Government Evidence of leadership and commitment in providing physical activity opportunities for all children and youth.

Allocated funds and resources for the implementation of physical activity promotion strategies and initiatives for all children and youth. Demonstrated progress through the key stages of public policy making (ie, policy agenda, policy formation, policy implementation, policy evaluation and decisions about the future).

\section{Data Analysis}

For analysis purposes, the 49 participating countries and regions were divided into 3 categories using the United Nations' HDI classification (low or medium, high, and very high HDI) described above. Sociodemographic data that are available online were compiled to identify the characteristics of the participating countries. Descriptive statistics (average grade and standard deviation) were calculated after converting categorical variables (letter grades) to interval variables (see corresponding numbers in Table 2), and the incomplete (INC) grades were converted into null values, which were treated as missing values. Averages were calculated by country, indicator, and category of HDI from the interval values, and the floor (the number rounded down) was converted back to a letter grade. Three scores were generated for the analysis: (1) overall score computed as the sum of interval values for all indicators, (2) behavioral score (the sum of Overall Physical Activity, Organized Sport and Physical Activity, Active Play, Active Transportation, and Sedentary Behaviors interval values), and (3) sources of influence score (the sum of Family and Peers, School, Community and Environment, and Government interval values). "INC" grades were removed, and the scores were reweighted accordingly (ie, the missing grade was replaced by the sum of the interval values divided by the number of letter grades included in the score). Categorical variables (letter grades) were grouped into 4 ("A-B," "C," "D-F," and "No Grade") based on the 
Table 2 Global Matrix 3.0 Grading Rubric

\begin{tabular}{lcc}
\hline Grade & Interpretation & Corresponding number for analysis \\
\hline A+ & $94 \%-100 \%$ & 15 \\
A & We are succeeding with a large majority of children and youth $(87 \%-93 \%)$ & 14 \\
A- & $80 \%-86 \%$ & 13 \\
B+ & $74 \%-79 \%$ & 12 \\
B & We are succeeding with well over half of children and youth $(67 \%-73 \%)$ & 11 \\
B- & $60 \%-66 \%$ & 10 \\
C+ & $54 \%-59 \%$ & 9 \\
C & We are succeeding with about half of children and youth $(47 \%-53 \%)$ & 8 \\
C- & $40 \%-46 \%$ & 7 \\
D+ & $34 \%-39 \%$ & 6 \\
D & We are succeeding with less than half but some children and youth $(27 \%-33 \%)$ & 5 \\
D- & $20 \%-26 \%$ & 4 \\
F & We are succeeding with very few children and youth $(<20 \%)$ & 2 \\
INC & Incomplete-insufficient or inadequate information to assign a grade & No grade \\
\hline
\end{tabular}

overall score. These categories were then used to rank countries by the letter grade/score and category level in scatter plots. Correlational analyses between the 10 common indicators and sociodemographic indicators were performed using Spearman's rank correlation coefficients. Only significant moderate to strong correlations were considered for the discussion in this analysis $(r \geq .30$ or $r \leq-.30, P<.05) .{ }^{29}$ Pairwise deletion was used to treat missing data (INC grades) in order to minimize the number of cases excluded from the analysis. All statistical analyses were performed, and maps were created in $\mathrm{R}$ version 3.4.1 (The $\mathrm{R}$ Foundation for Statistical Computing, Vienna, Austria). Several packages were loaded to extend base R including corrplot, ${ }^{30}$ ggplot $2,{ }^{31} \mathrm{UpSetR},{ }^{32}$ and VIM. ${ }^{33}$

\section{Results}

A total of 52 countries responded to the AHKGA and showed interest in participation in the Global Matrix 3.0, but only 51 fully registered on time, and later on 2 countries withdrew. Sociodemographic characteristics of the 49 countries participating in the Global Matrix 3.0 are presented in Table 3. The HDI scores ranged from 0.448 (Ethiopia) to 0.985 (Jersey). Ethiopia also scored the lowest for the following: growth national income per capita $(\$ 1523$ USD), mean years of schooling (2.6 y), global food security index (33.3), urban population percentage (17.2\%), and prevalence of people using improved drinking water sources (44\%). Qatar scored highest in the growth national income per capita (\$129,916 USD) and the gender inequality index (0.542). Public health expenditure in percentage of Gross Domestic Product was the highest in Sweden $(10.0 \%)$ and the lowest in Venezuela (1.5\%). Life expectancy at birth ranged from 53.1 years in Nigeria to 84.2 years in Hong Kong. Hong Kong also had the highest urban population percentage (100\%) and the highest population density (6987 peo$\mathrm{ple} / \mathrm{km}^{2}$ of land area). The lowest Gini index (least income inequality) was observed in Slovenia (25.4) and the highest (greatest income inequality) in Botswana (60.5).

The physical activity grades for the 10 common indicators are organized by country in alphabetical order (Table 4). A total of 490 grades, including 369 letter grades and 121 "INC" grades, were assigned by the 49 Report Card work groups. A "not applicable" grade was assigned to Active Transportation by Qatar's Report Card work group because of unsafe road conditions and a hot climate during most of the year. ${ }^{45}$ The grade count, number of "INC" grades, mean number grade, SD, and mean letter grade by indicator and group of indicators are presented in Table 5. The indicators with the lowest number of "INC" grades were Overall Physical Activity and Active Transportation $(n=2)$, whereas the indicator with the highest number of "INC" grades was Active Play $(n=29)$, followed by Physical Fitness $(n=27)$, and Family and Peers $(n=22)$. The mean letter grades ranged from " $D$ " for Overall Physical Activity to "C" for Organized Sport and Physical Activity, Active Transportation, School, Community and Environment, and Government. An average grade of "C-" was obtained for the behavioral indicators, "C" for the sources of influence indicators, and "C-" was the overall average grade for the 369 letter grades. The average letter grades by indicator and group of indicators for the low or medium, high, and very high HDI countries are presented in Table 6. For the low or medium HDI countries, an average of " $C$ " was obtained for the behavioral indicators and "D+" for the source of influence indicators, whereas for the very high HDI countries, an average of "D+" was obtained for the behavioral indicators and "C+" for the sources of influence indicators. For the high HDI countries, an average of "D+" was obtained for the behavioral indicators and for the sources of influence indicators. Overall, an average grade of "C-," "D+," and "C-" was obtained for the low and medium HDI countries, high HDI countries, and very high HDI countries, respectively.

A plot for the estimated overall score of each country from the 10 indicators is presented in Figure 1, and the behavioral and sources of influence scores are shown in Figures 2 and 3, respectively. Slovenia ranked first, whereas China ranked 49th for the overall score (full rankings are shown in Figure 1); Nepal ranked first, and Estonia ranked last for the behavioral score (complete rankings are shown in Figure 2); and Slovenia ranked first, whereas Venezuela ranked last for the source of influence score (see all country rankings in Figure 3). The Supplementary Material 1 (available online) presents the correlation coefficients and their associated $P$ values between and within the 10 core physical activity indicators and the sociodemographic indicators. It showed that there were no statistically significant relationships between the 


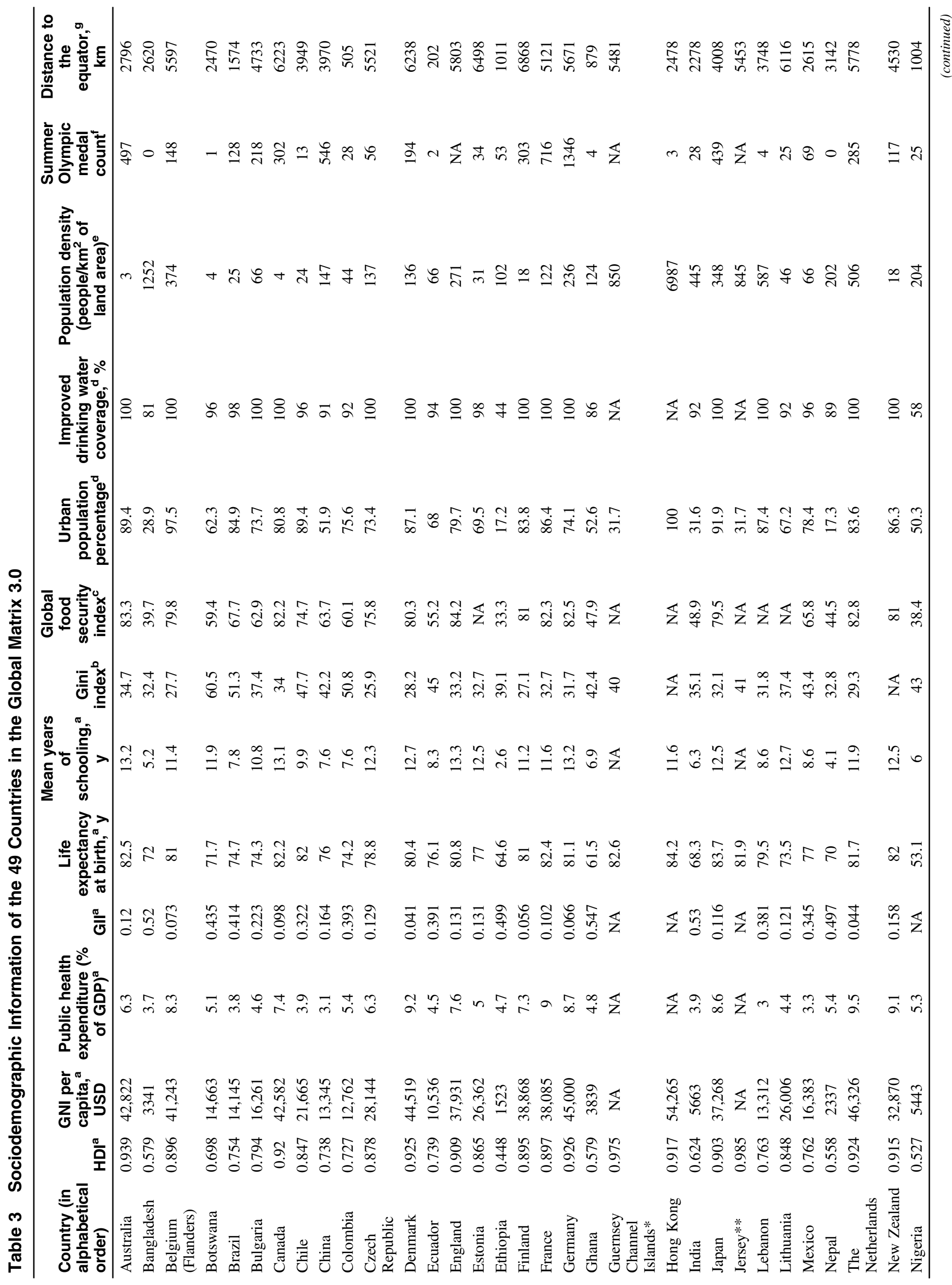




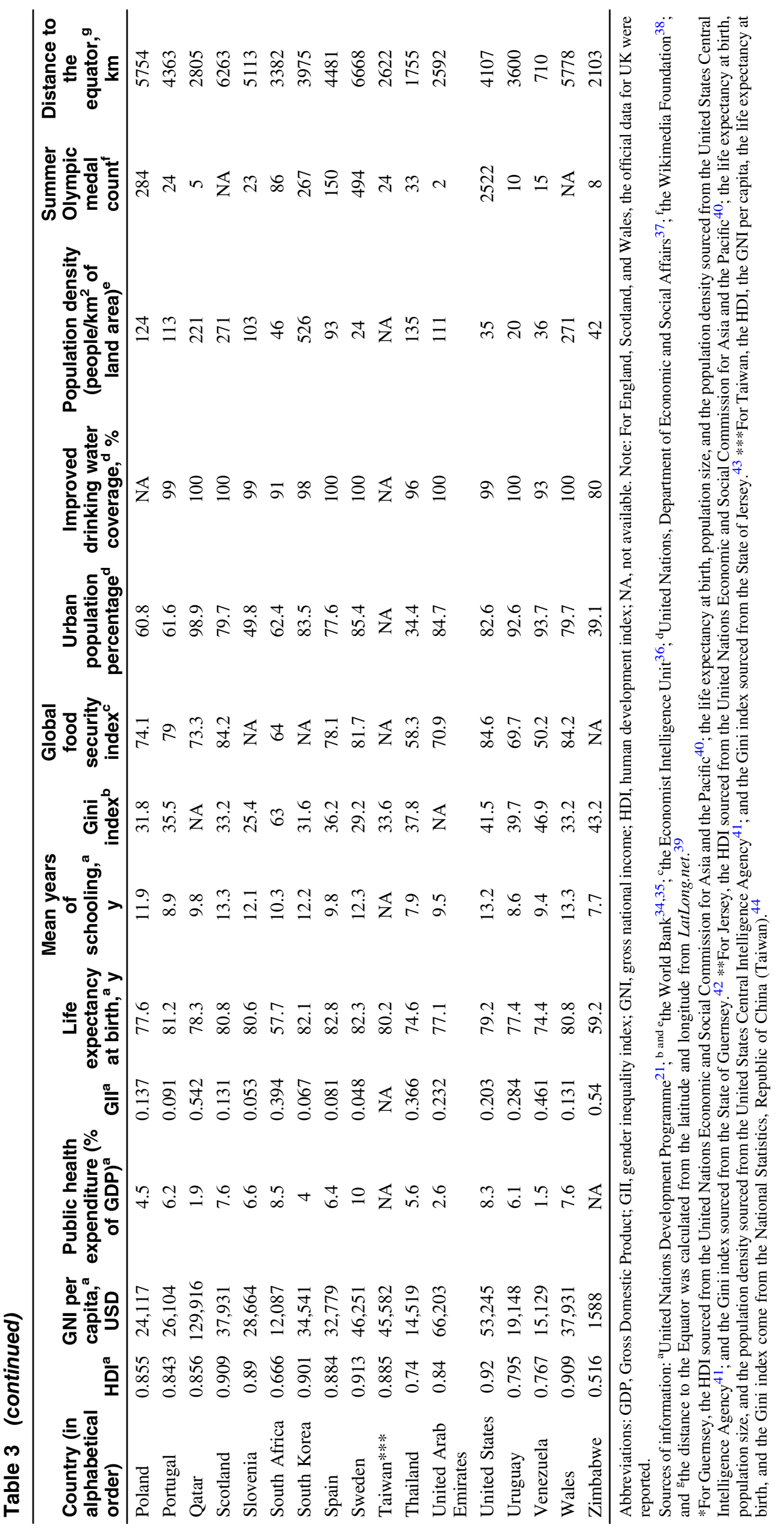




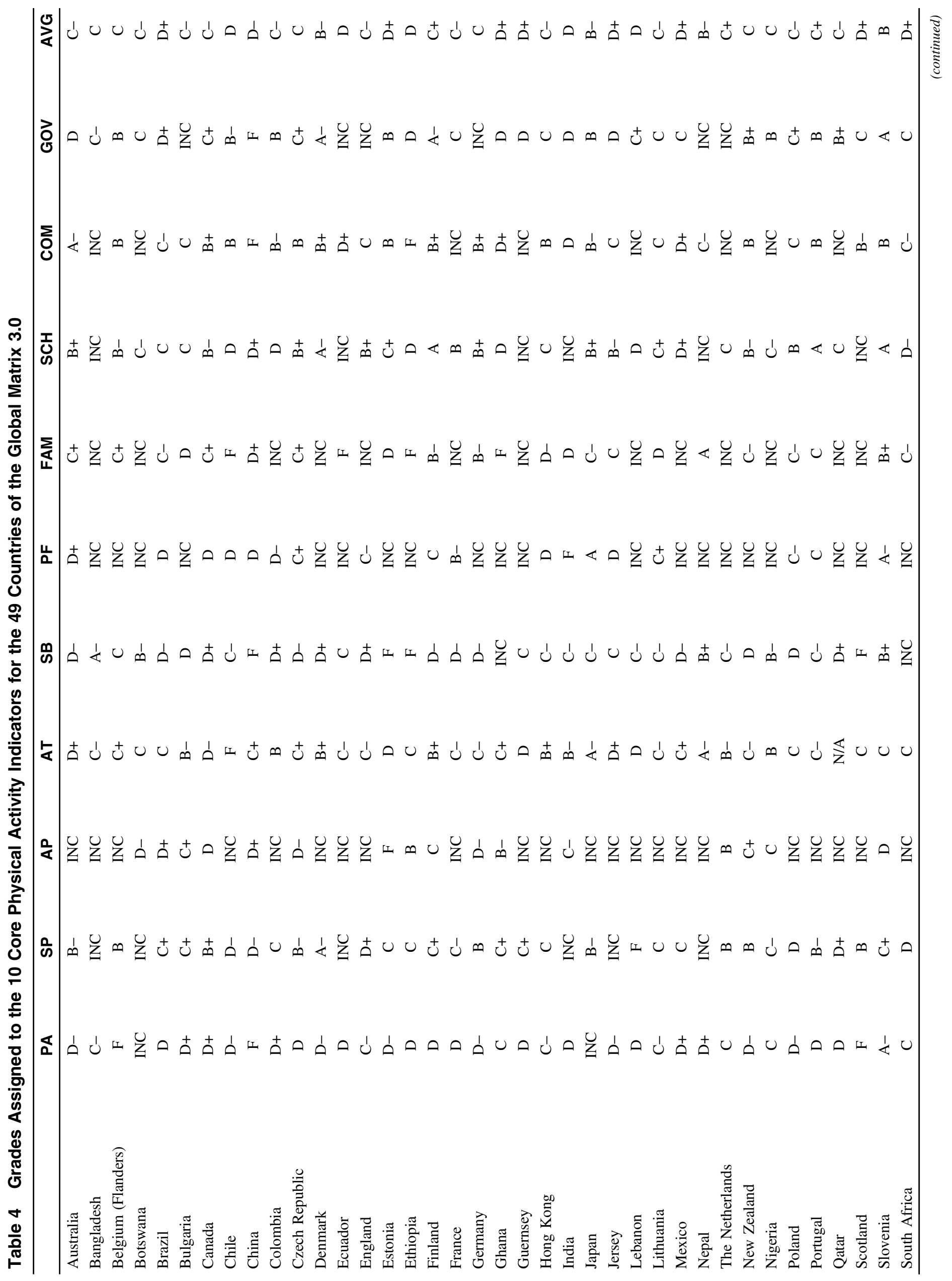




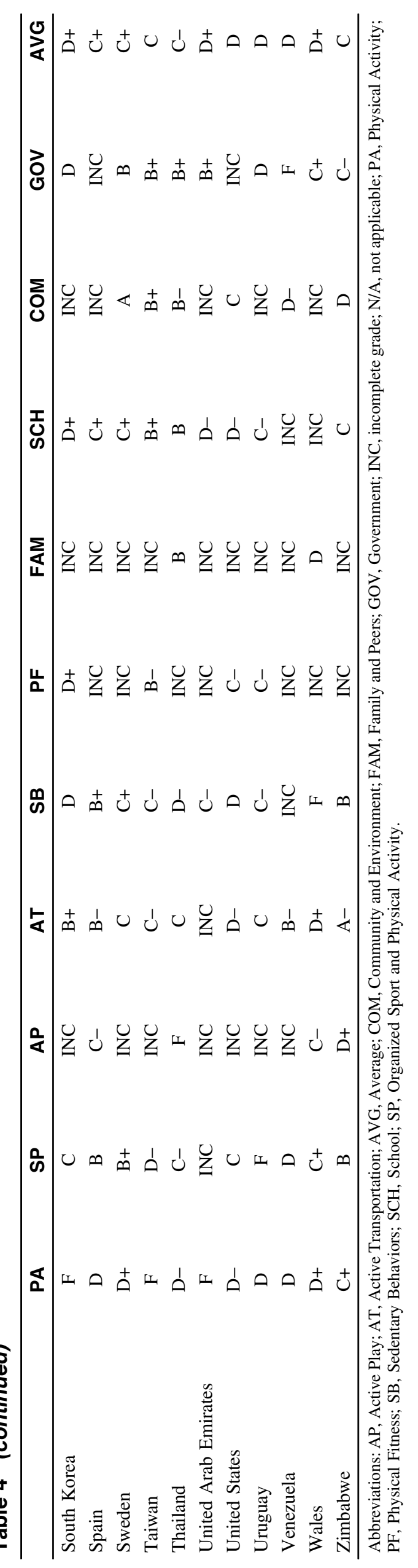


Table 5 Descriptive Statistics of the Grades by Indicator and Groups of Indicators for the 49 Countries of the Global Matrix 3.0

\begin{tabular}{lccccc}
\hline & Grade count & Incomplete grades & Mean number grade & SD & Mean letter grade \\
\hline Overall Physical Activity & 47 & 2 & 5.2 & 2.1 & $\mathrm{D}$ \\
Organized Sport and Physical Activity & 42 & 7 & 8.2 & 2.7 & $\mathrm{C}$ \\
Active Play & 20 & 29 & 6.5 & 2.7 & $\mathrm{D}+$ \\
Active Transportation & 47 & 2 & 8.3 & 2.5 & $\mathrm{C}$ \\
Sedentary Behaviors & 46 & 3 & 6.4 & 2.8 & $\mathrm{D}+$ \\
Physical Fitness & 22 & 27 & 7.1 & 2.9 & $\mathrm{C}-$ \\
Family and Peers & 27 & 22 & 7.0 & 3.1 & $\mathrm{D}+$ \\
School & 41 & 8 & 8.8 & 3.0 & $\mathrm{C}$ \\
Community and Environment & 36 & 13 & 8.9 & 3.0 & $\mathrm{C}$ \\
Government & 41 & 8 & 8.6 & 3.1 & $\mathrm{C}$ \\
Behavioral indicators & $\mathbf{4 9}$ & $\mathbf{0}$ & $\mathbf{7 . 0}$ & $\mathbf{1 . 6}$ & $\mathbf{C}-$ \\
Sources of influence indicators & $\mathbf{4 9}$ & $\mathbf{0}$ & $\mathbf{8 . 3}$ & $\mathbf{2 . 5}$ & $\mathbf{C}$ \\
All indicators & $\mathbf{4 9}$ & $\mathbf{0}$ & $\mathbf{7 . 5}$ & $\mathbf{1 . 6}$ & $\mathbf{C}-$ \\
\hline
\end{tabular}

Note: Behavioral indicators = average of Overall Physical Activity, Organized Sport and Physical Activity, Active Play, Active Transportation, and Sedentary Behavior indicator grades; sources of influence indicators = average of Family and Peers, School, Community and Environment, and Government indicator grades. Physical Fitness was not included in the behavioral indicators cluster. There are no missing grades for the bottom 3 rows (bolded) because these scores are adjusted for missing grades.

Table 6 Average Grades by Indicator and Group of Indicators for the 3 HDI Categories (Low or Medium, High, and Very High)

\begin{tabular}{lccc}
\hline & Low and medium HDI & High HDI & Very high HDI \\
\hline Overall Physical Activity & $\mathrm{C}-$ & $\mathrm{D}-$ & $\mathrm{D}-$ \\
Organized Sport and Physical Activity & $\mathrm{C}$ & $\mathrm{D}+$ & $\mathrm{C}+$ \\
Active Play & $\mathrm{C}-$ & $\mathrm{D}$ & $\mathrm{D}+$ \\
Active Transportation & $\mathrm{C}+$ & $\mathrm{C}$ & $\mathrm{C}-$ \\
Sedentary Behaviors & $\mathrm{C}+$ & $\mathrm{D}$ & $\mathrm{D}+$ \\
Physical Fitness & $\mathrm{F}$ & $\mathrm{D}$ & $\mathrm{C}-$ \\
Family and Peers & $\mathrm{D}+$ & $\mathrm{D}+$ & $\mathrm{C}-$ \\
School & $\mathrm{D}+$ & $\mathrm{C}-$ & $\mathrm{C}+$ \\
Community and Environment & $\mathrm{D}$ & $\mathrm{D}+$ & $\mathrm{B}-$ \\
Government & $\mathrm{C}-$ & $\mathrm{D}+$ & $\mathrm{C}+$ \\
Behavioral indicators & $\mathrm{C}$ & $\mathrm{D}+$ & $\mathrm{D}+$ \\
Sources of influence indicators & $\mathrm{D}+$ & $\mathrm{D}+$ & $\mathrm{C}+$ \\
All indicators & $\mathrm{C}-$ & $\mathrm{D}+$ & $\mathrm{C}-$ \\
\hline
\end{tabular}

Abbreviation: HDI, human development index. Note: Behavioral indicators = average of Overall Physical Activity, Organized Sport and Physical Activity, Active Play, Active Transportation, and Sedentary Behavior indicator grades; sources of influence indicators = average of Family and Peers, School, Community and Environment, and Government indicator grades. Physical Fitness was not included in the behavioral indicators cluster. There are no missing grades for the bottom 3 rows (bolded) because these scores are adjusted for missing grades.

Overall Physical Activity grade and the other core indicator grades with the exception of Sedentary Behaviors for which a statistically significant positive moderate correlation was observed $(r$ $=.39, P<.05)$. Finally, presented in the Supplementary Material 2 (available online) is the distribution of the grades ("A-B," "C," "D$\mathrm{F}$," or "INC" grades) for the 10 common indicators and the average grades per country. Slovenia was the only country out of 49 with a very high grade for Overall Physical Activity ("A-"), whereas most of the other countries had "D-F" $(n=38)$. A greater variability in grades was observed for Sedentary Behaviors, but poor grades ("DF") were observed in most of the very high HDI countries.

\section{Discussion}

As a result of the efforts of the Report Card work groups, the Global Matrix 3.0 allowed us to present physical activity-related indicators for children and youth assembled across 49 countries with varying levels of human development (9 low or medium HDI, 10 high HDI, and 30 very high HDI countries). While the average grades calculated for the indicators were all between " $\mathrm{D}$ " and " $\mathrm{C}$ " (Table 5), a great variety of grades was observed within and across the countries (Table 4), showing that every country is facing unique challenges and can learn from the successes or difficulties experienced by 


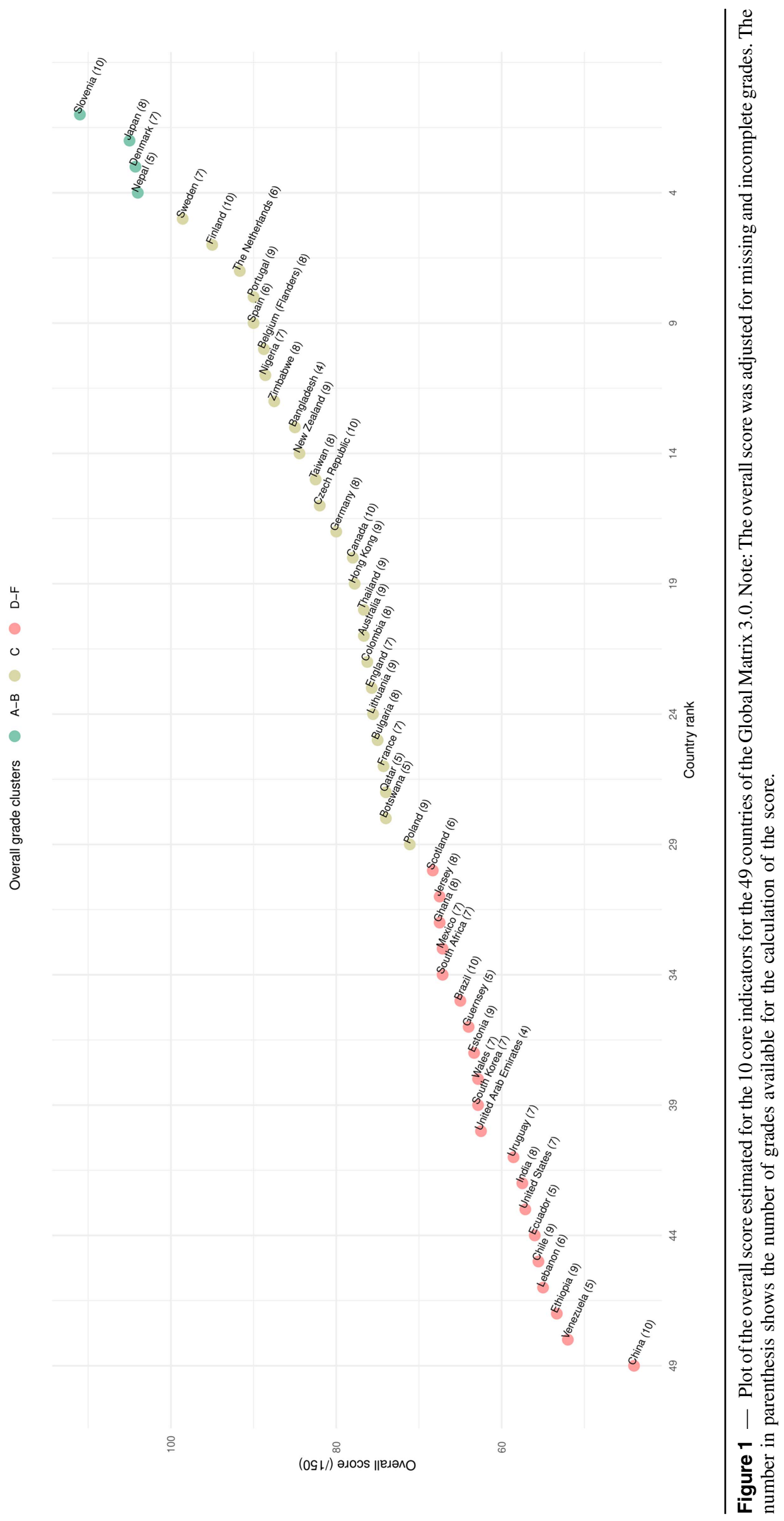




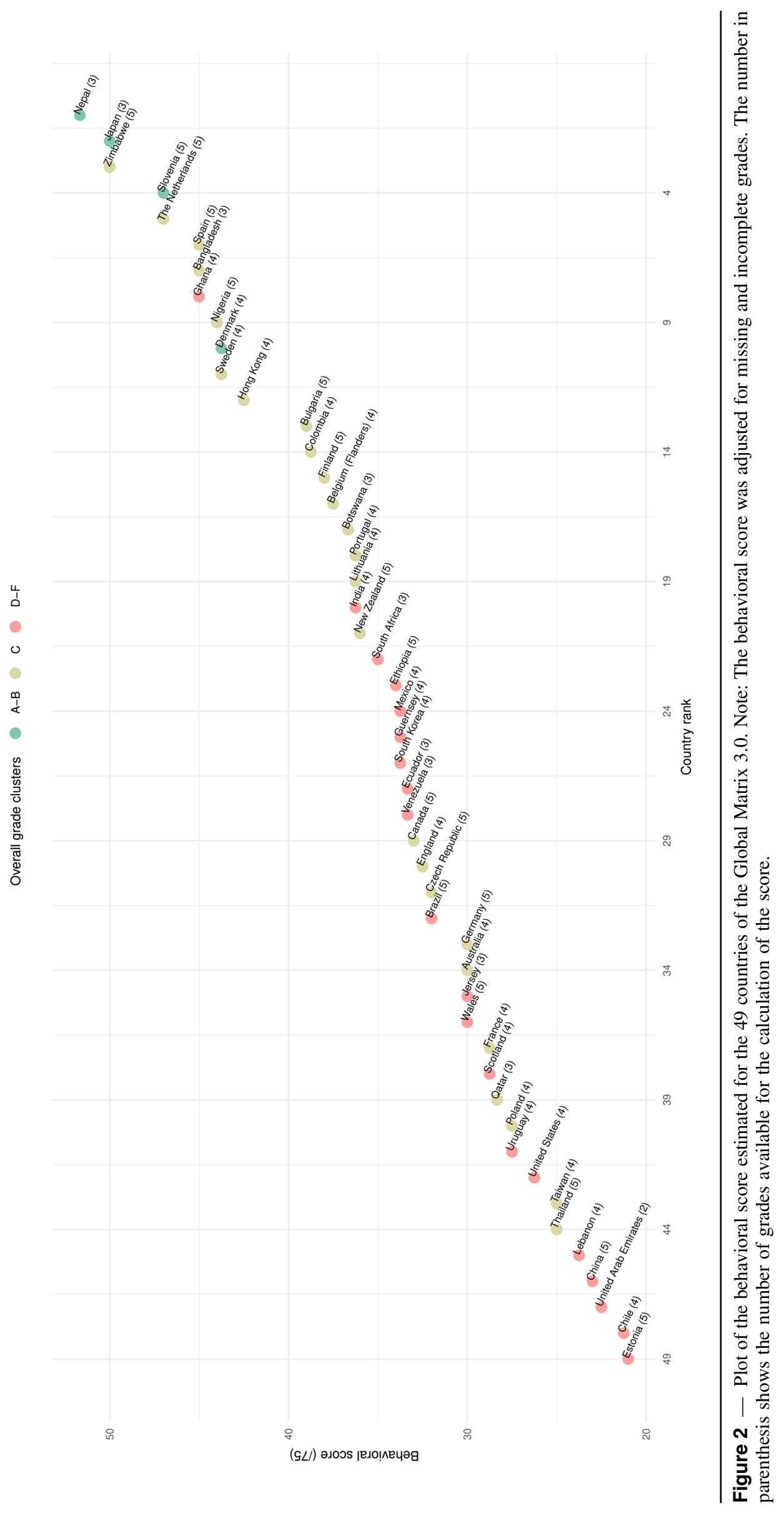




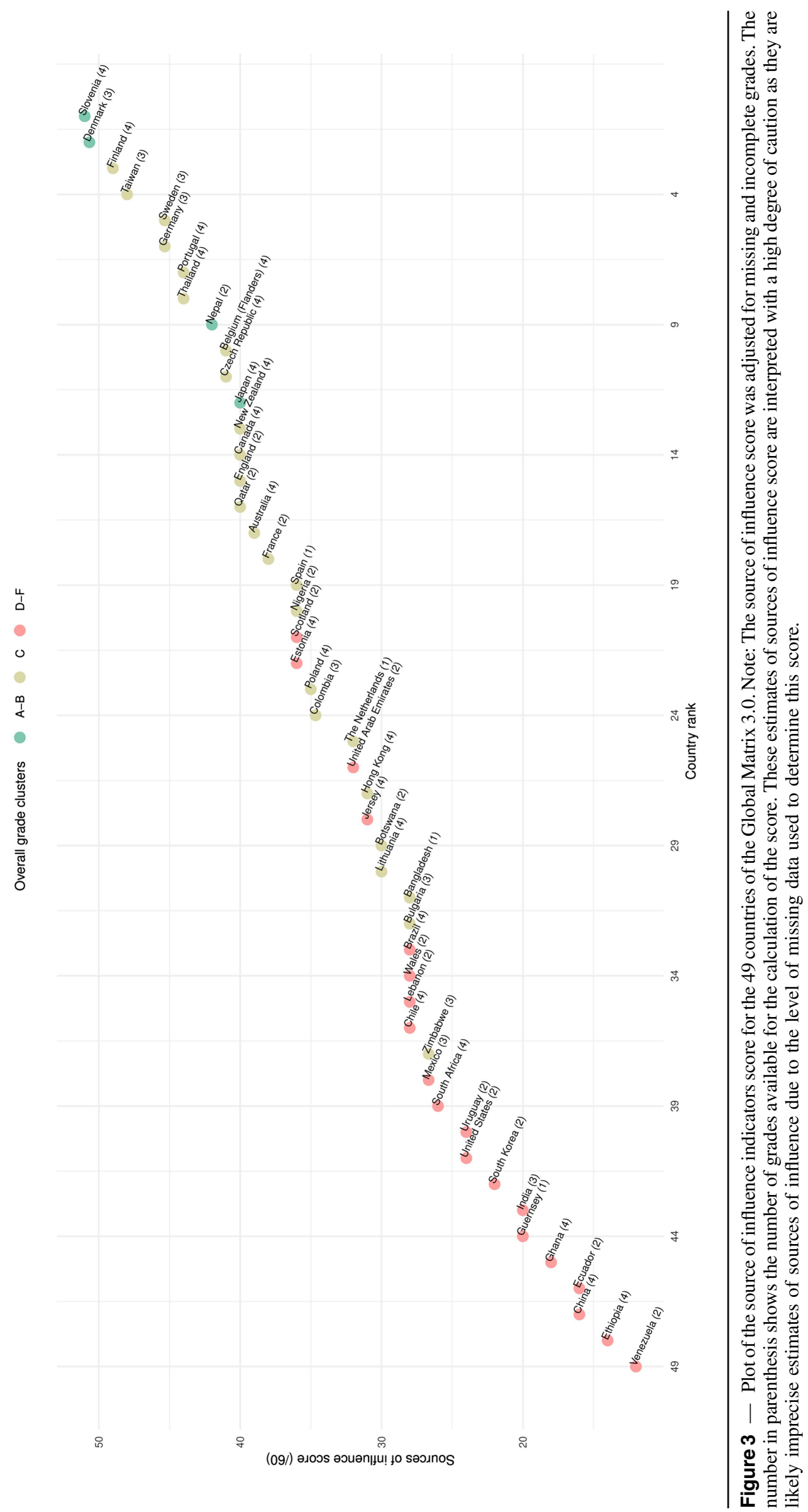


others. However, trends and comparable challenges identified across the physical activity indicators were identified within countries in the same HDI grouping despite their diverse sociodemographic, cultural, and geographical contexts. A discussion of the most and the least successful countries, followed by the interpretation of the findings from each indicator is presented in the following section, integrating the comparison of each HDI grouping.

\section{Most Successful Countries}

On the overall score, the very high HDI countries ranked generally higher than the low and medium, and high HDI countries (Figure 1), but this is not corroborated with results presented in Table 6 where the overall averages were the same for the low and medium HDI countries and the very high HDI countries. Slovenia obtained the best grade on average ("B"), followed by 2 other very high HDI countries (Japan and Denmark) that both obtained an average grade of "B-." The specifics of childhood physical activity in these 3 countries have already been discussed in the paper presenting the findings from the very high HDI countries. ${ }^{25}$ These 3 countries were also leading the ranking based on the sources of influence score (Figure 3). The ranking of the behavioral score was dominated by 2 low HDI countries (Nepal and Zimbabwe) and Japan (Figure 2). These rankings should be interpreted with caution given the large number of "INC" grades in their country Report Cards (2 in Japan, 3 in Denmark, 5 in Nepal, and 2 in Zimbabwe).

Nepal lacked data to inform 5 of the 10 indicators that were assigned an "INC" grade (Organized Sport and Physical Activity, Active Play, Physical Fitness, School, and Government). A "D+" was assigned to Overall Physical Activity based on a study that found that only $39.8 \%$ of children and youth accumulated at least 60 minutes of MVPA per day. Nevertheless, Nepal shared the best grade for Active Transportation with Japan and Zimbabwe ("A-"), obtained a high grade for Sedentary Behaviors ("B+") and also scored highest for the Family and Peers indicator ("A"). These grades were all informed by local studies focusing on adolescents. ${ }^{46}$ Nepal's good grades in many of the indicators including Active Transportation and Sedentary Behavior may be associated with low automobile dependency (eg, 7.12 vehicles for 1000 people in 2011) ${ }^{47}$ and minimal opportunities and access to screen-based recreational sedentary pursuits such as television and computer time and time spent using the Internet. Nepal had 5.30 televisions per 1000 people (as of 2003), 4.37 computer (as of 2004), and 4.51 Internet subscribers (as of 2012) for 1000 people. ${ }^{48}$ Due to the concurrent economic development in recent years in Nepal, the number of motorized vehicles is increasing rapidly with the expansion of road networks in rural areas, ${ }^{49}$ and potentially more people have access to screen devices. These findings should be interpreted with caution, but they do suggest that the situation can potentially be positive in Nepal in terms of child and youth's active transportation and sedentary behaviors at present. Yet, these favorable behaviors might be threatened by the economic growth and development, and these physical activity behaviors may be more a function of poor economic development than a freely chosen physical activity. Nonetheless, more good quality research with nationally representative data is needed to obtain more reliable estimates of the physical activity of children and youth and to inform the grades with "INC" grades.

Zimbabwe had the second-best grade for Overall Physical Activity ("C+") after Slovenia, with high grades for most of the behavioral indicators ("B" for Organized Sport and Physical Activity, "A-" for Active Transportation, and "B" for Sedentary
Behaviors); however, its sources of influence indicators were assigned grades between "D" and "C." In comparison with the previous Report Card in Zimbabwe, the grades for School, Community and Environment, and Government, have improved due to recent policy implementations and commitments made by the government to promote physical activity and nutritional status among Zimbabwean children and youth. ${ }^{50}$ However, there is need for caution in interpreting these grades as they were informed by limited and mostly unpublished data and expert opinion. Thus, more research is needed to obtain a reliable picture of the context of the physical activity level of children and youth in Zimbabwe.

\section{Least Successful Countries}

One low HDI country, Ethiopia, and 2 high HDI countries, Venezuela and China, were the least successful countries based on the overall and the source of influence score rankings (Figures 1 and 3). An average grade of "D-" was assigned to China, and an average grade of " $D$ " was assigned to Ethiopia and Venezuela. China's Report Card work group developed a national surveillance protocol to collect nationally representative data for China's 2018 Report Card. ${ }^{51}$ An " $F$ " was assigned to 4 indicators (Overall Physical Activity, Sedentary Behaviors, Community and Environment, and Government) and the remaining indicators were assigned grades between "D-" and "C+." The small proportions of 9- to 17-year-old children in China meeting the physical activity guidelines (13.1\%) or the sedentary behavior guidelines $(7.1 \%)$ are alarming. ${ }^{51}$ With an estimated population of 1.4 billion, ${ }^{52}$ China is the most populated country in the world, and the low prevalence estimates observed here suggest that the majority of a large number of children and youth (approximately 160 million of 10 - to 19 -y-olds in 2015$)^{52}$ are not engaging in sufficient amounts of MVPA to benefit their health. However, the high level of air pollution in China represents a major threat to the promotion of physical activity ${ }^{53}$ and is a potential barrier stopping the Chinese children and youth from exercising. The recent support from the Chinese Ministry of Education in conducting national surveillance of children physical activity is however encouraging, and hopefully this support will be expanded to future investments in the development of interventions and policies designed to increase physical activity opportunities at community and environment levels in China.

In Ethiopia, an "F" was assigned to Sedentary Behaviors, Family and Peers, and Community and Environment, and the grades for the remaining indicators ranged between " $D$ " and "C," with the exception of Active Play that was graded "B." These findings should be interpreted with caution as the grades were informed by estimates based on experts' opinion when data for an indicator were unavailable. ${ }^{54}$ This method, nevertheless, did allow the Report Card work group to present an initial broad picture of childhood physical activity in Ethiopia.

Venezuela is currently facing a humanitarian crisis related to its economic and sociopolitical situation. Between 2016 and 2017, marked increases in maternal mortality $(65 \%)$, infant mortality $(30 \%)$, and cases of malaria $(76 \%)$ were observed, and the estimated prevalence of severe malnutrition among children under 5 years of age increased from 10.2\% in February 2017 to $14.5 \%$ in September 2017. ${ }^{55}$ Nevertheless, a Report Card was developed, using published and unpublished national survey data, peerreviewed literature, government and nongovernment reports and online content, and meetings with experts working for governmental (municipal) and nongovernmental organizations. ${ }^{56}$ The Venezuelan Report Card work group assigned an "INC" grade to 
5 indicators: Active Play, Sedentary Behaviors, Physical Fitness, Family and Peers, and School. An "F" grade was assigned to the Government indicator while the remaining indicators were graded "D" or "D-," with the exception of Active Transportation which was graded "B-." Although there were no physical activity data available for those under 15 years of age, the work group found that the majority of adolescents older than 15 years do not engage in physical activity regularly. In addition, the high rates of crime were identified as a barrier to physical activity. Currently, no governmental efforts to ensure safety and promote an active lifestyle in Venezuela are being implemented. ${ }^{56}$

\section{Overall Physical Activity}

Only 2 countries assigned "INC" grades to the Overall Physical Activity indicator: Japan and Botswana. Slovenia, where more than $80 \%$ of children and youth aged between 6 and 19 years (according to subjective self-reported data), $88 \%$ of the 11-year-olds, and $66 \%$ of the 14-year-olds (according to objective data) were meeting the physical activity guidelines, was the only country reporting a high grade for this indicator ("A-"). ${ }^{57}$ For this indicator, the grades for the remaining countries ranged from "F" (Belgium, China, Scotland, South Korea, and Taiwan) to "C+" (Zimbabwe).

The Overall Physical Activity grade was informed by various types of data across countries: objective measurement with accelerometers or pedometers, self-report or proxy-report questionnaire, and expert opinion. Even among both the subjective and objective data, the methods varied substantially in terms of instruments, analysis, age range, sample size, and representativeness of samples. ${ }^{23,24,58}$ In addition, the available data in each country did not necessarily allow the Report Card work groups to use either of the recommended benchmarks (see Table 1) strictly when estimating the prevalence of physically active children and youth in their sample. ${ }^{23,24,58}$ For such reasons, the comparability of these results among the countries should be interpreted with caution.

Overall Physical Activity was the indicator with the lowest average grade: "D." A distinction was observed between the low and medium HDI countries and the 2 other HDI groupings. The average grade for the low and medium HDI countries was "C-," whereas both the high and very high HDI countries obtained an average of "D-," which could represent a difference of $14 \%$ to $26 \%$ in physical activity guidelines adherence ("D-" $=20 \%-26 \%$ vs "C -" 40\%-46\%). In accordance with this difference of grades, a significant low negative correlation was observed between the Overall Physical Activity indicator and several sociodemographic indicators including the HDI $(r=-.30, P<.05)$ and the growth national income per capita $(r=-.33, P<.05)$ (see Supplementary Material 1 [available online]). The present study provides new evidence showing that the situation regarding the physical activity of children and youth is a universal concern worldwide. This finding is consistent with the results from the previous Global Matrices ${ }^{16,19}$ and recent global estimates. ${ }^{11}$ Efforts should be made globally and collectively to develop standardized physical activity surveillance systems adapted to the national context of each country. Furthermore, developing effective strategies to increase physical activity opportunities for all should be a national public health priority in all countries regardless of the HDI background.

\section{Organized Sport and Physical Activity}

An "INC" grade was assigned for this indicator in 7 countries: Bangladesh, Botswana, Ecuador, India, Jersey, Nepal, and United
Arab Emirates. The Organized Sport and Physical Activity grades ranged from "F" (Lebanon and Uruguay) to "A-" (Denmark), with an average of "C." The benchmark recommended for this indicator was the "percentage of children and youth who participate in organized sport and/or physical activity programs" (Table 1), meaning that this indicator did not provide any information on the dose (ie, duration, frequency, intensity) of physical activity while participating in sport and organized physical activities, because few countries have such data. The grade for this indicator depends on the availability of organized sport opportunities and the availability of data reporting the prevalence of children and youth who have taken advantage of these opportunities. This grade was mostly informed by official reports from governmental and/or public institutions as well as self-reported surveys.

Organized Sport and Physical Activity was graded "C," "D+," and "C+" on average for the low and medium HDI countries, the high HDI countries, and the very high HDI countries, respectively. Among the 9 low and medium HDI countries, 5 assigned a letter grade to this indicator, which ranged from " $D$ " to "B," with an average of "C." In these countries, the organized sport opportunities corresponded mostly to school-based sports or sport teams and recreational sports organized by nongovernmental organizations and communities. Among the high HDI countries, the grades ranged from " $\mathrm{F}$ " to " $\mathrm{C}$ +" with an average of "D+." However, it is not possible to discern from the available data if these low grades correspond to the limited availability of organized sport opportunities (ie, distal correlates) or if there are barriers (eg, proximal correlates such as motivation, interest, time, or parental support) hindering children from participating while organized physical activities are readily available to them. In the very high HDI countries, with an average grade of "C+," Organized Sport and Physical Activity was the highest graded behavioral indicator. In these countries, organized sport opportunities are available to children and youth in various settings: sport club and federations, school-based sport teams and organized sport sessions, municipal sport programs, and sport programs offered by private businesses. In addition, significant low to moderate positive associations were observed between Organized Sport and Physical Activity and several sociodemographic indicators including HDI, life expectancy at birth, mean years of schooling, growth national income per capita, public health expenditure (\% of GDP), global food security index, improved drinking water coverage, and summer Olympic medal count, whereas significant low negative associations between this indicator and the 2 inequality indices (Gini index and gender inequality index) (see Supplementary Material 1 [available online]) were observed.

More research is needed to examine if affordable and appealing organized physical activity and sport opportunities are offered to all children and youth equally and equitably-across different age, gender, socioeconomic, ethnic, and special population groups-and to isolate the missing components of organized sports opportunities in each country. In addition, it would be interesting to add the measurement of the dose of physical activity associated with organized sports and physical activities in national physical activity surveillance systems. Filling these research and surveillance gaps is a necessary step toward the development of effective strategies to promote physical activity by increasing organized sport opportunities at the national level.

\section{Active Play}

Among the 20 countries that graded Active Play, this behavior was mostly measured by self- or proxy-report surveys, assessing the frequency or the time of active play/being active while 
playing, ${ }^{57,59-62}$ unstructured/unorganized active play, ${ }^{51,63-69}$ playing outdoors/outdoors activities/being outdoors, ${ }^{50,59,67,70-72}$ and/or was based on expert opinion. ${ }^{50,54,73,74} \mathrm{~A}$ definition for active play was proposed in a recent systematic review: "a form of gross motor or total body movement in which young children exert energy in a freely chosen, fun, and unstructured manner."75 A consensus definition, however, still needs to be internationally agreed upon and acknowledged to allow the development of standardized measurement tools for this indicator in varying age groups.

Active Play was the indicator with the most "INC" grades where 29 of 49 countries were unable to find available data to grade this indicator. The grades ranged from " $F$ " (Estonia and Thailand) to "B" (Ethiopia and The Netherlands) with an average of "D+." The average grades for the low and medium HDI countries, high HDI countries, and very high HDI countries were " $C-$," "D," and "D+," respectively. Given the amount of "INC" grades and the variability of the data used to inform the grades for the Active Play indicator, a valid comparison between the 3 HDI groupings was difficult. The low grades that were reported for this indicator aligned with the previous Global Matrices findings. ${ }^{16,19}$ Such low level of engagement in active play can potentially be explained by the low detection capacity of instruments utilized for its measurement. Active play during free time may be slowly disappearing in favor of screen time in developed countries or is replaced with chores or work in developing countries. Perception of the environment as unsafe can also be a potential barrier to this behavior. However, no relation was found between Active Play and the Community and Environment indicators (Supplementary Material 1 [available online]). Further research is needed for the development of a consensus definition, measurement instruments, surveillance systems, and to identify the barriers and facilitators of active play.

\section{Active Transportation}

Only 2 countries did not assign a letter grade to Active Transportation: Qatar (not applicable) and United Arab Emirates ("INC"). The average grade for this indicator was D+ and ranged from "F" (Chile) to "A-" (Japan, Nepal, and Zimbabwe). The recommended benchmark for this indicator was "the percentage of children and youth who use active transportation to get to and from places" (Table 1). For most of the countries, the grades were informed by data reporting the prevalence of children and/or youth actively commuting between home and school without information on different doses (ie, frequency, duration, intensity).

Active Transportation was the indicator with the highest average grade for the low and medium HDI countries ("C+") and for the high HDI countries ("C"). The average grade for the very high HDI countries was " $\mathrm{C}-$-" for this indicator. In 3 of the 4 very high HDI countries from Eastern Asia, very high grades were assigned to this indicator: Japan ("A-"), Hong Kong ("B+"), and South Korea ("B+"). These 3 countries share similar characteristics historically, culturally, and developmentally, ${ }^{76}$ and have shown a high percentage of urban population $(83.3 \%-100 \%$, Table 3$)$. A specific policy in Japan (limiting the distance between the children and youth homes and the public elementary and junior high schools $)^{77}$ and the high density of Hong Kong, Japanese, and South Korean cities, allow children and youth to live a short trip away from their school, which can minimize the use of cars or other forms of motorized vehicles, and promote active travel. ${ }^{25,78,79}$

High to very high grades for this indicator were also reported in several developing countries: Colombia ("B"), Nepal ("A-"),
Nigeria ("B"), Venezuela ("B-"), and Zimbabwe ("A-"), whereas poor grades were reported for 16 of the 30 very high HDI countries (with grades between " $F$ " and "C-"). However, no statistically significant relation was found between Active Transportation and Community and Environment indicators or the HDI in the correlational analysis. These findings suggest that, for developing countries, active transportation may be driven by a necessity (ie, no access to public or family/personal motorized transportation) instead of a choice, ${ }^{80}$ notwithstanding the safety of the environment or the long distance to commute. The measurement of the dose and the characteristics of children's and youth's active transportation internationally are necessary to identify the contribution of active transport to overall physical activity levels, as well as the facilitators and obstacles for this behavior in order to develop contextually adapted, effective ways to promote it. Strategies to improve safety conditions and to promote active transportation as a desirable mode of transport are required in order to maintain the high grades in the country's leading this indicator and to improve those that are lagging.

\section{Sedentary Behaviors}

Sedentary Behaviors is defined as "any waking behavior characterized by an energy expenditure $\leq 1.5$ metabolic equivalents, while in a sitting, reclining, or lying posture."1 Screen time, referring to time spent in screen-based behaviors, ${ }^{1}$ is often used as a proxy for sedentary behavior in research. Screen time can be performed while being sedentary or physically active ${ }^{1}$; however, this behavior has been shown to be associated with a variety of negative health outcomes among children and youth. ${ }^{81}$ This is why guidelines recommending limiting screen time to 2 hours daily for 5- to 17 year-olds were developed for the first time in Canada. ${ }^{82}$ Furthermore, it was also the reason why the benchmark for Sedentary Behaviors focused on screen time: "percentage of children and youth who meet the Canadian Sedentary Behaviour Guidelines (5to 17-year-olds: no more than 2 hours of recreational screen time per day)" (Table 1). However, the comparisons between the countries should be interpreted with caution because of the variability of the data that were used to inform the grades for this indicator. Several Sedentary Behaviors grades were partially or totally informed by data reporting time spent sitting or doing other sedentary activities that did not involve screens, and screen time cutoff points differing from the one in the benchmark (ie, $\leq 1 \mathrm{~h} / \mathrm{d},<2$ used hours/day, $\leq 3 \mathrm{~h} / \mathrm{d}$ ) were also used by some countries because of available data.

Three countries assigned an "INC" grade to Sedentary Behaviors: Ghana, South Africa, and Venezuela. The grades for this indicator ranged from " $F$ " (China, Estonia, Ethiopia, Scotland, and Wales) to "A-" (Bangladesh), with an average of "D+." These findings are consistent with international estimates reporting that at least two-thirds of the children exceed 2 hours of recreational screen time per day, ${ }^{83}$ although comparison may be limited by the heterogeneity in Sedentary Behaviors across countries. The low and medium HDI countries obtained an average of "C+" for this indicator, and only 2 of the 8 countries had a low grade: Ethiopia "F" and India "C-." The grades for the 6 remaining low and medium HDI countries ranged from "B" to "A-." The high and the very high HDI countries obtained an average grade of " $D$ " and "D+," respectively, for Sedentary Behaviors. In 36 out of the 39 high and very high HDI countries that graded this indicator, a low or very low grade (between "F" and "C-") was assigned. Regardless, the Sedentary Behaviors grades were not significantly related to HDI or to the other sociodemographic indicators except the mean 
years of schooling $(r=.31, P<.05)$ and the summer Olympic medal count $(r=-.57, P<.05)$ in the correlational analysis presented in the Supplementary Material 1 (available online).

The results presented here suggest that the situation concerning the childhood screen time is particularly concerning in high and very high HDI countries. The moderately good grades for Sedentary Behavior in the low and medium HDI countries are potentially threatened to decrease with their continuing economic growth and development, which may lead to increased access to electronic devices. Several interventions to reduce screen time have been developed and tested, and results from systematic reviews and meta-analyses reported that interventions to reduce children's screen time can have a small but significant effect. ${ }^{84}$ More research is necessary to accumulate more evidence on the effectiveness of specific interventions across different contexts and settings, but investment from public health institutions in high and very high HDI countries to support the implementation of these interventions in the child and youth population should become a priority.

\section{Physical Fitness}

Physical fitness represents the ability to perform daily activities with vigor, and the demonstration of traits and capacities that are associated with a lower risk of the premature development of diseases associated with physical inactivity. ${ }^{85}$ Cardiorespiratory endurance, muscular endurance, muscular strength, body composition, and flexibility are the health-related components of physical fitness. ${ }^{86}$ The Global Matrix 3.0 evaluated the Physical Fitness indicator for the first time, and 27 countries were unable to find available data to inform the grade for this indicator. In the remaining countries, the grade for Physical Fitness ranged from " $F$ " (India) to "A" (Japan), with an average of "C-." India was the only country from the low and medium HDI countries with a letter grade for this indicator. Among the 10 high HDI countries, only 4 had a letter grade reported for this indicator: Brazil ("D"), China ("D"), Colombia ("D-"), and Uruguay ("C--"), with an average of "D." A letter grade was assigned to 17 of the 30 very high HDI countries, ranging from "D" (Canada, Chile, Hong Kong, and Jersey) to "A" (Japan).

The benchmark for this indicator corresponded to the average percentile achieved on certain health-related physical fitness components based on the normative values published by Tomkinson et al (Table 1). ${ }^{27}$ However, many differences in the data informing the grades for this indicator were observed across countries in terms of number fitness indicators reported, normative value used, age range, and sample size. Given the amount of "INC" grades and variability in the data informing the grades for this indicator, the comparison between HDI groupings is difficult. Cardiorespiratory fitness (measured with the 20-m shuttle run test) was found to be associated with favorable indicators of adiposity and some indicators of cardiometabolic, cognitive, and psychosocial health in boys and girls from 32 countries in a recent systematic review. ${ }^{87}$ Therefore, cardiorespiratory fitness is an important indicator of current and future health among school-aged children and youth and can be used as a holistic indicator of population health in this age group. ${ }^{88}$ These findings highlight that global surveillance of physical fitness, which represents a simple and cost-effective assessment ${ }^{89}$ that could be integrated in physical education classes, should be a priority in all countries.

\section{Family and Peers}

The average grade for Family and Peers was the same among the 3 HDI groupings: "D+." An "INC" grade was assigned in
22 countries: 4 from the low and medium HDI group, 6 from the high HDI group, and 12 from the very high HDI group. The grades ranged from " $F$ " (Chile, Ecuador, Ethiopia, and Ghana) to "A" (Nepal). This amount of "INC" grades and the letter grades reported are consistent with the previous Global Matrices. ${ }^{16,19}$

Support from parents and peers is recognized as a correlate of physical activity in children and youth ${ }^{90}$; however, the nature of this relationship is complex and no valid and internationally recognized instrument exists to measure the influence of family and friends on children and youth's physical activity at present. This is why several benchmarks were proposed to capture a picture of the influence of Family and Peers (Table 1), and variability in the data used to inform the letter grades for this indicator was observed. The number of "INC" grades and the measurement variation for this indicator limit the comparison and the interpretation of the letter grades reported. However, these findings highlight the need for the development of standardized methods for the surveillance of this indicator in various settings and contexts.

\section{School}

Given the potentially significant amount of time that children and youth spend at school, this environment is a strategically important setting for the promotion of physical activity. Physical activity opportunities can be provided to children and youth in the school environment through physical education, lunch and recess breaks, in-class physical activities, and in intramural competitive and noncompetitive activities before or after school. ${ }^{91}$ However, school physical activity policies, if they exist at all, differ from one country to another and are not always mandatory.

The average grade for the School indicator was " $C$," and Report Card work groups in 8 countries could not assign a letter grade to this indicator (Bangladesh, Ecuador, Guernsey, India, Nepal, Scotland, Venezuela, and Wales). This indicator ranged from "D-" (South Africa, United Arab Emirates, and United States) to "A" (Finland, Portugal, and Slovenia). The average grade for this indicator was "D+" for the low and medium HDI countries. For the high HDI countries, School was the source of influence indicator that obtained the highest average grade ("C-"). The very high HDI countries obtained an average grade of " $\mathrm{B}-$," and it is worth noting that only medium to favorable grades (" $\mathrm{C}$ " to "A") were assigned to European countries (high and very high HDI counties included). The correlational analysis found a significant moderate association between the School indicator and 6 of the sociodemographic indicators: the HDI $(r=.53, P<.001)$, the mean years of schooling $(r=.51, P<.001)$, the Gini Index $(r=-.66$, $P<.001)$, the gender inequality index $(r=-.65, P<.001)$, the global food security index $(r=.52, P<.05)$, and the distance to equator $(r=.55, P<.001)$. In other words, the grades for School indicator increased as the HDI, the mean years of schooling, the food security, and the distance to the equator increased, and as the gender inequality index and the Gini index decreased. These findings align with the results of the Global Matrix 2.0. ${ }^{19}$

These findings further suggest that the quality and/or quantity of physical activity opportunities offered by the school are associated with the economic and development status of a given country. Cultural values attributed to sport and or physical activity can also be potential barriers or facilitators for this indicator. Quality school policies and programs related to physical activity for the 3 European countries leading this indicator (Finland, Portugal, and Slovenia) have already been highlighted elsewhere. ${ }^{19,25}$ While mandatory physical education classes are part of the school 
curriculum in most European countries for all school grades, it is only optional in some other countries from other parts of the world. For example, in the United States, the percentage of schools delivering mandatory physical education classes, in each grade, decrease from $97 \%$ in sixth grade to $42 \%$ in 12 th grade. ${ }^{92}$ In the United Arab Emirates, only 26\% of adolescents aged 13-17 years reported participating in physical education class on 3 or more days each week. ${ }^{93}$ In South Africa, $32 \%$ of children do not participate in school physical education classes, and no evidence of progress in the prioritization of physical education in the schools was found by the Report Card work group. ${ }^{94}$ Similarly, 2 consecutive Report Cards from South Korea highlighted that the emphasis on important subjects (eg, math, science) for university admission overrides the importance of physical activity in the Korean education system. ${ }^{95,96}$ Although the new 2018 Korean national curriculum requires schools to provide mandatory physical education, it is regarded as a minor subject and, with progressing school grades, it is devalued and neglected. ${ }^{97}$

Evidence showed that adding more time to academic or curricular subjects by taking time away from physical education programs was found not to enhance academic achievement in the corresponding academic subjects and to be potentially detrimental to health. ${ }^{98}$ Conversely, allocating more time to physical activity from other subjects can improve the time children spend engaging in MVPA without the risk of "hindering students' academic achievement." 98 The measure of the dose of physical activity occurring in school should also be added to the national surveillance systems globally to identify the needs in terms of policies and interventions aimed at promoting physical activity at school. In addition, the Global Matrix 3.0 findings suggest that developing interventions or programs targeting schools in low and medium HDI countries should be a priority on the international public health agenda.

\section{Community and Environment}

The Community and Environment indicator obtained an average grade of "C" and had 13 "INC" grades. The grades ranged from "D-" (Venezuela) to "A" (Sweden). The low and medium HDI countries obtained an average of "D," and the high HDI countries obtained a "D+." Among the very high HDI countries, the Community and Environment indicator was the indicator with the highest average grade ("B-"). The correlational analysis (see Supplementary Material 1 [available online]) found a positive significant moderate to strong association between the Community and Environment indicator and the HDI $(r=.73, P<.001)$, the life expectancy at birth $(r=.74, P<.001)$, mean years of schooling $(r=.64, P<.001)$, growth national income per capita $(r=.80$, $P<.001)$, public health expenditure $(r=.67, P<.001)$, global food security index $(r=.71, P<.001)$, urban population percentage $(r=.51, P<.001)$, improved drinking water coverage $(r=.77$, $P<.001)$, and distance to equator $(r=.58, P<.001)$; and a negative significant moderate to strong association was found with the Gini Index $(r=-.62, P<.001)$ and gender inequality index $(r=-.83$, $P<.001)$. These findings aligned with the results of the previous Global Matrices ${ }^{16,19}$ and reinforce the importance of income and gender equity for the physical activity and overall population health.

Characteristics of the built environment are recognized as a potential source of influence on the physical activity levels of children and youth. ${ }^{99-101}$ Given that a great number of characteristics could potentially influence the physical activity of children and youth, numerous benchmarks were proposed to evaluate this indicator. A great variability of data was used to inform the grades for this indicator, limiting direct comparisons between countries. Yet, the significant correlations suggest that despite the variability of data available that was used to inform the grade for this indicator, the Report Card work groups assigned a grade that was coherent with the characteristics of the environment. The available evidence from this indicator suggests that the characteristics of the built environment potentially influencing the physical activity of children and youth are already favorable in the very high HDI countries, and that an increase of the grades for this indicator is expected with the further development of low, medium, and high HDI countries.

\section{Government}

The average grade for the Government grade was " $\mathrm{C}$," and an "INC" grade was assigned in 8 countries. With an average of " $\mathrm{C}-$," Government was the source of influence indicator with the highest average in low and medium HDI countries. The high HDI countries obtained an average of "D+," and the very high HDI countries a "C+." The highest grades for this indicator were reported in high and very high HDI countries. The correlation analysis found significant moderate associations between the Government indicator and the growth national income per capita $(r=.48, P<.01)$, public health expenditure $(r=.48, P<.01)$, Gini index $(r=-.45, P<.01)$, gender inequality index $(r=-.45, P<.01)$, improved water coverage $(r=.44, P<.01)$, and distance to the equator $(r=.31, P<.01)$.

Similar to the Community and Environment indicator, a government can potentially influence the physical activity level of children and youth in multiple ways. As there is no official standardized method to evaluate this influence, the recommended benchmark for this indicator focused on evidence of leadership and commitment, allocated funds and resources, and demonstrated progress for the promotion of physical activity opportunities for all children and youth (Table 1). As a result, the grades were informed by very different types of data across countries, and the Report Card work groups did not always have access to relevant quantitative data and, therefore, graded this indicator mostly based on expert opinion when required.

The low behavioral grades that were reported broadly in the Global Matrix 3.0 suggest that the government's efforts and physical activity policies are not reflected in an increase in the prevalence of MVPA among children and youth, and/or that there is an implementation gap between the strategy level (source of influence indicators) and individual level (behavioral indicators). More research is needed globally to identify the potential barriers for the engagement of children at the country and community level, in different contexts and settings.

\section{Integrated Discussion}

Overall, the average grades obtained for each indicator were low, and a small variation was observed within the 10 indicators (" $D$ " to "C"). More diversity was observed between the 3 HDI groupings, between the countries, and within each country. Higher behavioral grades and lower source of influence grades were generally observed in the low and medium HDI countries, whereas the opposite was observed for the very high HDI countries, and generally low average grades were reported for each indicator in the high HDI countries. It is important to highlight that the overall concerning levels of physical activity and sedentary behavior 
among children and youth may be indicative of a global crisis. Moreover, this phenomenon may get worse with the economic growth and development of low, medium, and high HDI countries, and very high rates of noncommunicable diseases can be anticipated when the current generation of children and youth reaches adulthood unless a major shift to a more active lifestyle happens soon. Strategies to mitigate the projected reduction in habitual physical activity in developing countries should learn from countries who have already navigated the physical activity transition. ${ }^{102}$

\section{Strengths and Limitations}

A major limitation of this study was the amount of missing data to inform the grades, resulting in a total of 121 "INC" grades of the 490 grades in total. In addition, the great variability of the data (eg, in terms of benchmark, measurement instrument, age range, sample size, quality, quantity) that were used to inform the grades was observed between the countries for each indicator, and in many cases, the available data did not permit the Report Card work groups to align strictly with the recommended benchmarks. Furthermore, a loss of information potentially occurred when translating original prevalence data to a letter grade, as letter grades provide less information than continuous variables. Consequently, all the comparisons, correlations, and interpretations involving the grades are limited and should be interpreted with caution, as 2 identical grades for the same indicator can capture 2 very different contexts from one country to another. Excluding the countries with insufficient data and imposing to assign "INC" grades every time the data did not fit exactly with the benchmarks for each indicator could have been an alternative strategy. But this approach would have significantly reduced the number of countries and grades included in the Global Matrix 3.0 and the relevance of conducting international comparisons. Despite the limitations of the underlying data, this encyclopedia of global information of the physical activity of children and youth represents the richest source of such information compiled to date.

Another limitation of the Global Matrix 3.0 was the lack of representativeness of some areas of the world and some specific child and youth populations. Indeed, the participation of a total of 49 countries or nations meant that most of the countries around the world were still missing. In particular, the South Pacific islands nations, where the average body mass indices for child and youth were estimated to be the highest of the world, ${ }^{103}$ are missing, as well as countries from Central Asia and North Africa. Additionally, children and youth with disabilities were not explicitly included in this analysis while the Global Matrix process could help to identify and circulate best-practice strategies in terms of physical activity promotion for these specific populations. ${ }^{19}$ Furthermore, the disparities and inequities across gender, socioeconomic status, or urban versus rural dwelling were mentioned in only a handful of Report Card articles; thus, they were not analyzed or discussed in this study.

The correlation analyses showed that the Overall Physical Activity grade had a moderate positive association with Sedentary Behaviors and was not statistically related with the 8 remaining indicators (Supplementary Material 1 [available online]). This can potentially be the consequence of the aforementioned limitations, but another hypothesis may explain this absence of relation. The absence of statistically significant correlations between Overall Physical Activity and Organized Sport and Physical Activity, Active Play, and Active Transportation may have resulted from the fact that the physical activity dose related to these indicators was not measured. The absence of a relation between the source of influence indicators and Overall Physical Activity may suggest that a favorable physical and social environment is not enough to induce individual engagement in sufficient amounts of MVPA. In addition, the absence of choice (ie, children who have no other options than using active transportation regardless of safety and any other characteristics of the source of influence), child labor, and the cultural values attributed to physical activity and sports were not evaluated in this study.

Major strengths of this work include the large number of countries who adopted the harmonized Report Card development process and the quantity of data used to inform an international, comprehensive, and meaningful comparison of physical activity behaviors of children and youth. In addition, the AHKGA facilitated and supported the development of Report Cards from 49 countries, by providing the Report Card work groups from each participating country with a platform to capture, synthesize, interpret, and publish the findings with autonomy. With the 49 countries participating and the 10 common indicators being graded by each participating country, the Global Matrix 3.0 represents the most comprehensive and meaningful comparison of the contexts of the physical activity of children and youth. In addition, the identification and discussion of major trends concerning the characteristics of the physical activity of children and youth among the 3 HDI groupings were realized for the first time. ${ }^{23-25}$ Finally, major research and surveillance gaps, including the need for the development and the international adoption of standardized methods to conceptualize and measure the ten indicators, were identified and highlighted for each indicator in the present paper.

The launch of the Global Matrix 3.0 is the result of a tremendous amount of work by the AHKGA Executive Committee members and from the physical activity experts and stakeholders from all over the world for the past 15 months. The 49 Report Cards were developed by supported and unsupported work groups of professionals who acknowledge the importance of a healthy and active lifestyle for the present and the future of the children and youth worldwide. The involvement of governmental and nongovernmental organizations in the development of some of the national Report Cards is a potential sign of a growing interest in the childhood physical inactivity crisis, and it is hoped that this awareness will be translated into support for the development of effective interventions increasing physical activity opportunities for all. In addition, the development of a national Report Card of physical activity for children and youth and the participation in the Global Matrix initiative were found to contribute to raising awareness on the childhood physical inactivity issue nationally and internationally, building capacity within participating countries, and potentially influencing the creation of physical activity opportunities in the future. ${ }^{23}$ It is hoped that more physical activity experts and stakeholders will rally with AHKGA members to pursue and expand the Global Matrix initiative and will help fill the identified gap in physical activity research and surveillance. The AHKGA, with the contribution of its international network of researchers, will be available to offer guidance to the countries willing to develop national surveillance of physical activity and policies or interventions promoting physical activity among children and youth.

\section{Conclusions}

A comprehensive summary of the grades, comparison, and interpretation of 10 physical activity indicators among 49 countries were presented in this Global Matrix 3.0 paper. Higher behavioral 
grades and lower source of influence grades were generally observed in the low and medium HDI countries, whereas lower behavioral grades and higher source of influence grades were observed for the very high HDI countries, and generally low average grades for each indicator in the high HDI countries. International research and surveillance gaps were also identified by the Global Matrix 3.0 and the Report Card initiatives. Internationally collaborative and collective efforts are needed to redirect the persisting trend of low physical activity and high sedentary behavior among children and youth worldwide. Strategic public investments to implement effective interventions within families, communities, and schools to increase physical activity opportunities are needed. It is hoped that researchers and relevant stakeholders will collaborate internationally to address the research and surveillance gaps highlighted in this paper and expand the Global Matrix initiative to include more countries.

\section{Acknowledgments}

The authors would like to acknowledge the then Active Healthy Kids Canada (now ParticipACTION) for developing the Report Card methodology, and the AHKGA Executive Committee for modifying and standardizing the benchmarks and grading rubric and leading this international initiative. We are grateful for all the hard work by each participating country's Report Card Work Group and Leaders and all other members of their Report Card Committees. We also want to thank Evan Turner for his work on the compilation of the sociodemographic characteristics of the participating countries.

\section{References}

1. Tremblay MS, Aubert S, Barnes JD, et al. Sedentary Behavior Research Network (SBRN)-Terminology Consensus Project process and outcome. IntJ Behav Nutr Phys Act. 2017;14(1):75. PubMed ID: 28599680 doi:10.1186/s12966-017-0525-8

2. World Health Organization. Mortality and burden of disease attributable to selected major risks. 2009:70. http://www.who.int/healthinfo/ global_burden_disease/GlobalHealthRisks_report_full.pdf. Accessed April 4, 2018.

3. World Health Organization. WHO. Global recommendation on physical activity for health. 2010. http://www.who.int/dietphysicalactivity/ factsheet_recommendations/en/. Accessed January 11, 2018.

4. Bull FC, Gauvin L, Bauman A, Shilton T, Kohl HW, Salmon A. The Toronto charter for physical activity: a global call for action. J Phys Act Health. 2010;7:421-422. https://pdfs.semanticscholar.org/4b34/ 24f9139dbf473ac035737ca53ac94c633639.pdf. Accessed April 5, 2018.

5. Janssen I, LeBlanc AG. Systematic review of the health benefits of physical activity and fitness in school-aged children and youth. Int $J$ Behav Nutr Phys Act. 2010;7(1):40. doi:10.1186/1479-5868-7-40

6. Biddle SJ, Asare M. Physical activity and mental health in children and adolescents: a review of reviews. Br J Sports Med. 2011;45(11): 886-895. PubMed ID: 21807669 doi:10.1136/bjsports-2011-090185

7. Strauss RS, Rodzilsky D, Burack G, Colin M. Psychosocial correlates of physical activity in healthy children. Arch Pediatr Adolesc Med. 2001;155(8):897. PubMed ID: 11483116 doi:10.1001/archpedi.155. 8.897

8. Poitras VJ, Gray CE, Borghese MM, et al. Systematic review of the relationships between objectively measured physical activity and health indicators in school-aged children and youth. Appl Physiol
Nutr Metab. 2016;41(6 suppl 3):S197-S239. doi:10.1139/apnm2015-0663

9. Donnelly JE, Hillman CH, Castelli D, et al. Physical activity, fitness, cognitive function, and academic achievement in children: a systematic review. Med Sci Sports Exerc. 2016;48(6):1197-1222. PubMed ID: 27182986 doi:10.1249/MSS.0000000000000901

10. Esteban-Cornejo I, Tejero-Gonzalez CM, Sallis JF, Veiga OL. Physical activity and cognition in adolescents: a systematic review. J Sci Med Sport. 2015;18(5):534-539. PubMed ID: 25108657 doi:10.1016/J.JSAMS.2014.07.007

11. Sallis JF, Bull F, Guthold R, et al. Progress in physical activity over the Olympic quadrennium. Lancet. 2016;388(10051):1325-1336. PubMed ID: 27475270 doi:10.1016/S0140-6736(16)30581-5

12. Kremer P, Elshaug C, Leslie E, Toumbourou JW, Patton GC, Williams J. Physical activity, leisure-time screen use and depression among children and young adolescents. J Sci Med Sport. 2014;17(2):183187. PubMed ID: 23648221 doi:10.1016/J.JSAMS.2013.03.012

13. McMahon EM, Corcoran P, O'Regan G, et al. Physical activity in European adolescents and associations with anxiety, depression and well-being. Eur Child Adolesc Psychiatry. 2017;26(1):111-122. PubMed ID: 27277894 doi:10.1007/s00787-016-0875-9

14. Blair SN, Cheng Y, Holder SJ. Is physical activity or physical fitness more important in defining health benefits? Med Sci Sports Exerc. 2001;33(6):S379-S399. https://journals.lww.com/acsmmsse/Fulltext/2001/06001/Is_physical_activity_or_physical_fitness_ more.7.aspx. Accessed July 15.

15. Telama R, Yang X, Viikari J, Välimäki I, Wanne O, Raitakari O. Physical activity from childhood to adulthood: a 21-year tracking study. Am J Prev Med. 2005;28(3):267-273. PubMed ID: 15766614 doi:10.1016/J.AMEPRE.2004.12.003

16. Tremblay MS, Gray CE, Akinroye K, et al. Physical activity of children: a global matrix of grades comparing 15 countries. J Phys Act Health. 2014;11(suppl 1):S113-S125. doi:10.1123/jpah.2014-0177

17. Colley RC, Brownrigg M, Tremblay MS. A model of knowledge translation in health. Health Promot Pract. 2012;13(3):320-330. PubMed ID: 22447666 doi:10.1177/1524839911432929

18. Tremblay MS, Barnes JD, Bonne JC. Impact of the Active Healthy Kids Canada Report card: a 10-year analysis. J Phys Act Health. 2014;11(suppl 1):S3-S20. doi:10.1123/jpah.2014-0167

19. Tremblay MS, Barnes JD, González SA, et al. Global matrix 2.0: report card grades on the physical activity of children and youth comparing 38 countries. J Phys Act Health. 2016;13(11 suppl 2): S343-S366. doi:10.1123/jpah.2016-0594

20. Active Healthy Kids Global Alliance. About us: Active Healthy Kids Global Alliance. 2016. https://www.activehealthykids.org/about-us/. Accessed May 27, 2018.

21. United Nations Development Programme. Human Development Index (HDI). Human development reports. 2017. http://hdr.undp.org/en/ content/human-development-index-hdi. Accessed April 7, 2018.

22. Land KC. The human development index: objective approaches (2). In: Wolfgang Glatzer, Laura Camfield, Valerie Møller, Mariano Rojas, ed. Global Handbook of Quality of Life. Dordrecht, The Netherlands: Springer; 2015:133-157. doi:10.1007/978-94-017-9178-6_7

23. Manyanga T, Barnes JD, Abdeta C, et al. The indicators of physical activity among children and youth in nine countries with low and medium human development indices: a global matrix 3.0 paper. $J$ Phys Act Health. 2018;15(suppl 2):S274-S283. doi:10.1123/jpah. 2018-0370

24. González SA, Barnes JD, Abi Nader P, et al. Report card grades on the physical activity of children and youth from 10 countries with high human development index-global matrix 3.0. J Phys Act Health. 2018;15(suppl 2):S284-S297. doi:10.1123/jpah.2018-0391 
25. Aubert S, Barnes JD, Aguilar-Farias N, et al. Report card grades on the physical activity of children and youth comparing 30 very high human development index countries. J Phys Act Health. 2018;15(suppl 2):S298-S314. doi:10.1123/jpah.2018-0431

26. Tremblay MS, Carson V, Chaput JP, et al. Canadian 24-hour movement guidelines for children and youth: an integration of physical activity, sedentary behaviour, and sleep. Appl Physiol Nutr Metab. 2016;41(6 suppl 3):S311-S327. doi:10.1139/apnm-2016-0151

27. Tomkinson GR, Carver KD, Atkinson F, et al. European normative values for physical fitness in children and adolescents aged 9-17 years: results from 2779165 Eurofit performances representing 30 countries [ahead of print]. Br J Sports Med. 2017. doi:10.1136/ bjsports-2017-098253

28. González SA, Triana CA, Abaunza C, et al. Results from Colombia's 2018 report card on physical activity for children and youth. $J$ Phys Act Health. 2018;15(suppl 2):S335-S337. doi:10.1123/jpah.20180507

29. Mukaka M. A guide to appropriate use of correlation coefficient in medical research. Malawi Med J. 2012;24(3):69-71. https://www.ajol. info/index.php/mmj/article/view/81576. Accessed August 23, 2018.

30. Wei T, Simko V, Levy M, Xie Y, Jin Y, Zemla J. Visualization of a correlation matrix: corrplot. 2017. https:/github.com/taiyun/ corrplot. Accessed July 5, 2018.

31. Wickham H. ggplot2: Elegant Graphics for Data Analysis. Vol 77. 2nd ed. New York, NY: Springer-Verlag; 2009.

32. Conway JR, Lex A, Gehlenborg N. UpSetR: an R package for the visualization of intersecting sets and their properties. Bioinformatics. 2017;33(18):2938-2940. PubMed ID: 28645171 doi:10.1093/ bioinformatics/btx364

33. Kowarik A, Templ M. Imputation with the R Package VIM. J Stat Softw. 2016;74(7):1-16. doi:10.18637/jss.v074.i07

34. The World Bank. GINI index (World Bank estimate) data. 2017. https://data.worldbank.org/indicator/SI.POV.GINI?view=map. Accessed April 7, 2018.

35. The World Bank. Population density (people per sq. km of land area) data. 2016. https://data.worldbank.org/indicator/EN.POP.DNST. Accessed June 27, 2018.

36. The Economist Intelligence Unit. Global food security index: overview. 2017. https://foodsecurityindex.eiu.com/Index. Accessed June 27, 2018.

37. United Nations Department of Economic and Social Affairs. Social indicators/population growth and distribution. 2012. https://unstats. un.org/unsd/demographic/products/socind/. Accessed June 27, 2018.

38. Wikimedia Foundation. All-time Olympic Games medal table. 2018. https://en.wikipedia.org/wiki/All-

time_Olympic_Games_medal_table. Accessed August 10, 2018.

39. Latlong.net. Latitude and longitude finder on map get coordinates. 2012. https://www.latlong.net/. Accessed August 10, 2018.

40. Hastings DA. Filling gaps in the human development index: findings for Asia and the Pacific. 2009. https://www.unescap.org/sites/ default/files/wp-09-02.pdf. Accessed July 17, 2018.

41. The United States Central Intelligence Agency. The World Factbook, guide to country profiles. 2018. https://www.cia.gov/library/ publications/resources/the-world-factbook/docs/profileguide.html. Accessed July 17, 2018.

42. State of Guernsey. Guernsey household income. 2015. https://www. gov.gg/CHttpHandler.ashx?id=110715\&p=0. Accessed July 17, 2018.

43. State of Jersey. Jersey household income distribution 2014/15. 2015. https://www.gov.je/SiteCollectionDocuments/Government\%20and\% 20administration/R\%20Income\%20Distribution\%20Survey\%20Report \%202014-15\%2020151112\%20SU.pdf. Accessed July 17, 2018.
44. National Statistics Republic of China (Taiwan). Report on the survey of family income and expenditure. 2017. https://eng.stat.gov.tw/ct. asp?xItem $=3417 \&$ CtNode $=1596 \& m p=5$. Accessed June 28, 2018.

45. Ibrahim I, Al Hammadi E, Sayegh S, et al. Results from Qatar's 2018 report card on physical activity for children and youth. $J$ Phys Act Health. 2018;15(suppl 2):S400-S401. doi:10.1123/jpah.2018-0502

46. Subedi N, Paudel S, Nepal S, Karki A. Results from Nepal's 2018 report card on physical activity for children and youth. J Phys Act Health. 2018;15(suppl 2):S386-S387. doi:10.1123/jpah.2018-0512

47. Macro Economy Meter. Vehicles per 1000 people-Nepal. ND. http://mecometer.com/whats/nepal/vehicles-per-thousand-people/. Accessed August 16, 2018.

48. NationMaster.com. Nepal Media Stats. 2018. http://www. nationmaster.com/country-info/profiles/Nepal/Media. Accessed August 16, 2018.

49. My Republica. 3.1 million motor vehicles on Nepali roads: DoTM. 2018. https://myrepublica.nagariknetwork.com/news/3-1-millionmotor-vehicles-on-nepali-roads-dotm/. Accessed August 16, 2018.

50. Manyanga T, Munambah NE, Mahachi CB, Makaza D, Mlalazi TF. Results from Zimbabwe's 2018 report card on physical activity for children and youth. J Phys Act Health. 2018;15(suppl 2):S433S435. doi:10.1123/jpah.2018-0520

51. Liu Y, Tang Y, Cao ZB, et al. Results from China's 2018 report card on physical activity for children and youth. J Phys Act Health. 2018;15(suppl 2):S333-S334. doi:10.1123/jpah.2018-0455

52. United Nations. World population prospects—population divisionUnited Nations. 2017. https://esa.un.org/unpd/wpp/Download/ Standard/Population/. Accessed August 17, 2018.

53. Lü J, Liang L, Feng Y, et al. Air pollution exposure and physical activity in china: current knowledge, public health implications, and future research needs. Int $J$ Environ Res Public Health. 2015;12(11):14887-14897. doi:10.3390/ijerph121114887

54. Abdeta C, Teklemariam Z, Deksisa A, Abera E. Results from Ethiopia's 2018 report card on physical activity for children and youth. J Phys Act Health. 2018;15(suppl 2):S353-S354. doi:10. 1123/jpah.2018-0457

55. Human Rights Watch. World Report 2018: Venezuela Human Rights Watch. 2018. https://www.hrw.org/world-report/2018/ country-chapters/venezuela. Accessed August 17, 2018.

56. Herrera-Cuenca M, Méndez-Pérez B, Landaeta-Jiménez M, et al. Results from Venezuela's 2018 report card on physical activity for children and youth. J Phys Act Health. 2018;15(suppl 2):S427S429. doi:10.1123/jpah.2018-0467

57. Sember V, Morrison SA, Jurak G, et al. Results from Slovenia's 2018 report card on physical activity for children and youth. J Phys Act Health. 2018;15(suppl 2):S404-S405. doi:10.1123/jpah.20180542

58. Aubert S, Aucouturier J, Ganière C, et al. Results from France's 2018 report card on physical activity for children and youth. J Phys Act Health. 2018;15(suppl 2):S360-S362. doi:10.1123/jpah.20180511

59. Bhawra J, Chopra P, Harish R, Mohan A. Results from India's 2018 report card on physical activity for children and youth. $J$ Phys Act Health. 2018;15(suppl 2):S373-S374. doi:10.1123/jpah.2018-0475

60. Demetriou Y, Hebestreit A, Reimers AK, et al. Results from Germany's 2018 report card on physical activity for children and youth. J Phys Act Health. 2018;15(suppl 2):S363-S365. doi:10. 1123/jpah.2018-0538

61. Takken T, de Jong N. Results from the Netherlands's 2018 report card on physical activity for children and youth. J Phys Act Health. 2018;15(suppl 2):S388-S389. doi:10.1123/jpah.2018-0513 
62. Smith M, Ikeda E, Hinckson E, et al. Results from New Zealand's 2018 report card on physical activity for children and youth. $J$ Phys Act Health. 2018;15(suppl 2):S390-S392. doi:10.1123/jpah.20180463

63. Gába A, Rubín L, Badura P, et al. Results from the Czech Republic's 2018 report card on physical activity for children and youth. J Phys Act Health. 2018;15(suppl 2):S338-S340. doi:10.1123/jpah.20180508

64. Nyawornota VK, Luguterah A, Sofo S, Aryeetey R. Results from Ghana's 2018 report card on physical activity for children and youth. J Phys Act Health. 2018;15(suppl 2):S366-S367. doi:10.1123/jpah. 2018-0459

65. Silva DAS, Christofaro DGD, de Moraes Ferrari GL, et al. Results from Brazil's 2018 report card on physical activity for children and youth. J Phys Act Health. 2018;15(suppl 2):S323-S325. doi:10. 1123/jpah.2018-0421

66. Saonuam P, Rasri N, Pongpradit K, Widyastari DA, Katewongsa P. Results from Thailand's 2018 report card on physical activity for children and youth. J Phys Act Health. 2018;15(suppl 2):S417S418. doi:10.1123/jpah.2018-0465

67. Barnes JD, Cameron C, Carson V, et al. Results from Canada's 2018 report card on physical activity for children and youth. $J$ Phys Act Health. 2018;15(suppl 2):S328-S330. doi:10.1123/jpah.2018-0454

68. Mäestu E, Kull M, Mooses K, et al. Results from Estonian's 2018 report card on physical activity for children and youth. $J$ Phys Act Health. 2018;15(suppl 2):S350-S352. doi:10.1123/jpah.2018-0456

69. Kämppi K, Aira A, Halme N, et al. Results from Finland's 2018 report card on physical activity for children and youth. $J$ Phys Act Health. 2018;15(suppl 2):S355-S356. doi:10.1123/jpah.2018-0510

70. Mileva B. Results from Bulgaria's 2018 report card on physical activity for children and youth. J Phys Act Health. 2018;15(suppl 2): S326-S327. doi:10.1123/jpah.2018-0422

71. Roman-Viñas B, Zazo F, Martínez-Martínez J, Aznar-Laín S, SerraMajem L. Results from Spain's 2018 report card on physical activity for children and youth. J Phys Act Health. 2018;15(suppl 2):S411S412. doi:10.1123/jpah.2018-0464

72. Edwards LC, Tyler R, Blain D, et al. Results from Wales' 2018 report card on physical activity for children and youth. J Phys Act Health. 2018;15(suppl 2):S430-S432. doi:10.1123/jpah.2018-0544

73. Tladi DM, Monnaatsie M, Shaibu S, Sinombe G. Results from Botswana's 2018 report card on physical activity for children and youth. J Phys Act Health. 2018;15(suppl 2):S320-S322. doi:10. 1123/jpah.2018-0420

74. Akinroye KK, Adeniyi AF. Results from Nigeria's 2018 report card on physical activity for children and youth. J Phys Act Health. 2018;15(suppl 2):S393-S394. doi:10.1123/jpah.2018-0514

75. Truelove S, Vanderloo LM, Tucker P. Defining and measuring active play among young children: a systematic review. J Phys Act Health. 2017;14(2):155-166. PubMed ID: 27775475 doi:10.1123/jpah.20160195

76. Lee EY, Yi KJ, Walker GJ, Spence JC. Preferred leisure type, value orientations, and psychological well-being among East Asian Youth. Leis Sci. 2017;39(4):355-375. doi:10.1080/01490400.2016. 1209139

77. Mori N, Armada F, Willcox DC. Walking to school in Japan and childhood obesity prevention: new lessons from an old policy. Am J Public Health. 2012;102(11):2068-2073. PubMed ID: 22994195 doi:10.2105/AJPH.2012.300913

78. Oh JW, Lee E, Lim J, et al. Results from South Korea's 2018 report card on physical activity for children and youth. J Phys Act Health. In press;15(suppl 2):S409-S410. doi:10.1123/jpah.2018-0518
79. Huang WY, Wong SHS, Sit CHP, et al. Results from Hong Kong's 2018 report card on physical activity for children and youth. $J$ Phys Act Health. 2018;15(suppl 2):S370-S372. doi:10.1123/jpah.20180468

80. Sarmiento OL, Lemoine P, Gonzalez SA, et al. Relationships between active school transport and adiposity indicators in school-age children from low-, middle- and high-income countries. Int J Obes Suppl. 2015;5(suppl 2):S107-S114. doi:10.1038/ijosup. 2015.27

81. Carson V, Hunter S, Kuzik N, et al. Systematic review of sedentary behaviour and health indicators in school-aged children and youth: an update. Appl Physiol Nutr Metab. 2016;41(6 suppl 3):S240-S265. doi:10.1139/apnm-2015-0630

82. Tremblay MS, LeBlanc AG, Janssen I, et al. Canadian sedentary behaviour guidelines for children and youth. Appl Physiol Nutr Metab. 2011;36(1):59-64. PubMed ID: 21326378 doi:10.1139/ H11-012

83. Atkin AJ, Sharp SJ, Corder K, van Sluijs EMF. International Children's Accelerometry Database (ICAD) collaborators. prevalence and correlates of screen time in youth. Am J Prev Med. 2014;47(6):803-807. PubMed ID: 25241193 doi:10.1016/j. amepre.2014.07.043

84. Biddle SJH, Petrolini I, Pearson N. Interventions designed to reduce sedentary behaviours in young people: a review of reviews. $\mathrm{Br} \mathrm{J}$ Sports Med. 2014;48(3):182-186. PubMed ID: 24347578 doi:10. 1136/bjsports-2013-093078

85. Pate RR. The evolving definition of physical fitness. Quest. 1988;40(3):174-179. doi:10.1080/00336297.1988.10483898

86. Caspersen CJ, Powell KE, Christenson GM. Physical activity, exercise, and physical fitness: definitions and distinctions for health-related research. Public Health Rep. 1985;100(2): 126-131.

87. Lang JJ, Belanger K, Poitras V, Janssen I, Tomkinson GR, Tremblay MS. Systematic review of the relationship between $20 \mathrm{~m}$ shuttle run performance and health indicators among children and youth. $J$ Sci Med Sport. 2018;21(4):383-397. PubMed ID: 28847618 doi:10. 1016/j.jsams.2017.08.002

88. Lang JJ, Tomkinson GR, Janssen I, et al. Making a case for cardiorespiratory fitness surveillance among children and youth. Exerc Sport Sci Rev. 2018;46(2):66-75. doi:10.1249/JES. 0000000000000138

89. Lang JJ, Tremblay MS, Léger L, Olds T, Tomkinson GR. International variability in $20 \mathrm{~m}$ shuttle run performance in children and youth: who are the fittest from a 50-country comparison? A systematic literature review with pooling of aggregate results. Br J Sports Med. 2018;52(4):276. PubMed ID: 27650256 doi:10.1136/bjsports2016-096224

90. Sterdt E, Liersch S, Walter U. Correlates of physical activity of children and adolescents: a systematic review of reviews. Health Educ J. 2014;73(1):72-89. doi:10.1177/001789691 2469578

91. Hills AP, Dengel DR, Lubans DR. Supporting public health priorities: recommendations for physical education and physical activity promotion in schools. Prog Cardiovasc Dis. 2015;57(4):368-374. PubMed ID: 25269062 doi:10.1016/J.PCAD.2014.09.010

92. Katzmarzyk PT, Denstel KD, Beals K, et al. Results from the United States 2018 report card on physical activity for children and youth. $J$ Phys Act Health. 2018;15(suppl 2):S422-S424. doi:10.1123/jpah. 2018-0476

93. Paulo MS, Nauman J, Abdulle A, et al. Results from the United Arab Emirates' 2018 report card on physical activity for children and 
youth. J Phys Act Health. 2018;15(suppl 2):S419-S421. doi:10. 1123/jpah.2018-0543

94. Draper CE, Tomaz SA, Bassett SH, Burnett C. Results from South Africa's 2018 report card on physical activity for children and youth. J Phys Act Health. 2018;15(suppl 2):S406-S408. doi:10.1123/jpah. 2018-0517

95. Song Y, Yang HI, Lee EY, et al. Results from South Korea's 2016 report card on physical activity for children and youth. $J$ Phys Act Health. 2016;13(11 suppl 2):S274-S278. doi:10.1123/jpah.20160402

96. Oh JW, Lee EY, Lim J, Lee SH, Jin Y, Oh B. Results from South Korea's 2018 report card on physical activity for children and youth. J Exerc Sci Fit. In press.

97. Lee KC, Cho SM. The Korean national curriculum for physical education: a shift from edge to central subject. Phys Educ Sport Pedagog. 2014;19(5):522-532. doi:10.1080/17408989.2014. 915299

98. Trudeau F, Shephard RJ. Physical education, school physical activity, school sports and academic performance. Int J Behav Nutr Phys Act. 2008;5(1):10. doi:10.1186/1479-5868-5-10
99. Sallis JF, Owen N, Fisher E, Sallis JF, Owen N, Fisher E. Ecological models of health behavior. In: Glanz K, Rimer BK, Viswanath K. ed. Health Behavior: Theory, Research, and Practice. 4th ed. JosseyBass; 2015:43-64.

100. Saelens BE, Sallis JF, Black JB, Chen D. Neighborhood-based differences in physical activity: an environment scale evaluation. Am J Public Health. 2003;93(9):1552-1558. PubMed ID: 12948979 doi:10.2105/AJPH.93.9.1552

101. Sallis JF, Johnson MF, Calfas KJ, Caparosa S, Nichols JF. Assessing perceived physical environmental variables that may influence physical activity. Res Q Exerc Sport. 1997;68(4):345-351. PubMed ID: 9421846 doi:10.1080/02701367.1997.10608015

102. Katzmarzyk PT, Mason C. The physical activity transition. J Phys Act Health. 2009;6(3):269-280. PubMed ID: 19564654 doi:10. 1123/jpah.6.3.269

103. Abarca-Gómez L, Abdeen ZA, Hamid ZA, et al. Worldwide trends in body-mass index, underweight, overweight, and obesity from 1975 to 2016: a pooled analysis of 2416 population-based measurement studies in 128.9 million children, adolescents, and adults. Lancet. 2017;390(10113):2627-2642. doi:10.1016/S0140-6736(17)32129-3 